\title{
LONG-TIME ASYMPTOTICS OF THE ONE-DIMENSIONAL DAMPED NONLINEAR KLEIN-GORDON EQUATION
}

\author{
RAPHAËL CÔTE, YVAN MARTEL, AND XU YUAN
}

\begin{abstract}
For the one-dimensional nonlinear damped Klein-Gordon equation $\partial_{t}^{2} u+2 \alpha \partial_{t} u-\partial_{x}^{2} u+u-|u|^{p-1} u=0 \quad$ on $\mathbb{R} \times \mathbb{R}$,

with $\alpha>0$ and $p>2$, we prove that any global finite energy solution either converges to 0 or behaves asymptotically as $t \rightarrow \infty$ as the sum of $K \geq 1$ decoupled solitary waves. In the multi-soliton case $K \geq 2$, the solitary waves have alternate signs and their distances are of order $\log t$.
\end{abstract}

\section{INTRODUCTION}

1.1. Main results. We consider the one-dimensional nonlinear focusing damped Klein-Gordon equation

$$
\partial_{t}^{2} u+2 \alpha \partial_{t} u-\partial_{x}^{2} u+u-f(u)=0, \quad(t, x) \in \mathbb{R} \times \mathbb{R}, \quad f(u)=|u|^{p-1} u,
$$

with $\alpha>0$ and $p>2$. It follows from standard arguments that the Cauchy problem for (1.1) is locally well-posed in the energy space $H^{1} \times L^{2}$ (see references in \$2.1). Moreover, the existence of solutions blowing up in finite time is well-known, [1]. Denote $F(u)=\frac{1}{p+1}|u|^{p+1}$. Defining the energy of a solution $\vec{u}=\left(u, \partial_{t} u\right)$ by

$$
E(\vec{u})=\frac{1}{2} \int\left\{\left(\partial_{t} u\right)^{2}+\left(\partial_{x} u\right)^{2}+u^{2}-2 F(u)\right\} \mathrm{d} x,
$$

it holds formally

$$
E\left(\vec{u}\left(t_{2}\right)\right)-E\left(\vec{u}\left(t_{1}\right)\right)=-2 \alpha \int_{t_{1}}^{t_{2}}\left\|\partial_{t} u(t)\right\|_{L^{2}}^{2} \mathrm{~d} t .
$$

It is also well-known that up to sign and translation, the only stationary solution of (1.1) is the solitary wave $(Q, 0)$, where $Q$ is the explicit ground state

$$
Q(x)=\left(\frac{p+1}{2 \cosh ^{2}\left(\frac{p-1}{2} x\right)}\right)^{\frac{1}{p-1}},
$$

which solves the equation

$$
Q^{\prime \prime}-Q+Q^{p}=0 \quad \text { on } \mathbb{R} \text {. }
$$

Remarkable results and techniques developed in [1, 2, 11, 13, 14, provide information on the long-time asymptotic behavior of global solutions of (1.1) and of higher dimensional variants of this model for suitable nonlinearities. From techniques in 1, 2], all global solutions are proved to be bounded in the energy space (see Theorem 2.2). From [11 and the concentration-compactness principle as stated in 13, 14, any global solution either converges to zero in the energy space, or decomposes along a subsequence of time into a sum of decoupled ground states (see Theorem 2.3 and Remark 2.5). For space dimension $2 \leq N \leq 6$, it is proved in [1] that any global radially symmetric solution converges either to 0 or to a single

2010 Mathematics Subject Classification. 35L71 (primary), 35B40, 37K40.

Y. M. and X. Y. thank IRMA, Université de Strasbourg, for its hospitality. 
solitary wave as $t \rightarrow \infty$. In [6], in space dimension $1 \leq N \leq 5$ and for energy subcritical nonlinearities, global solutions of the damped Klein-Gordon equation containing two solitary waves are described and classified.

The objective of the present article is to complement those works by describing precisely the asymptotic behavior as $t \rightarrow \infty$ of any global solution of (1.1). The choice of considering the one-dimensional model is discussed in Remark 2.6.

Theorem 1.1. For any global solution $\vec{u} \in C\left([0, \infty), H^{1} \times L^{2}\right)$ of (1.1), one of the following three scenarios occurs:

Vanishing: $\vec{u}(t)$ converges exponentially to 0 in $H^{1} \times L^{2}$ as $t \rightarrow \infty$.

Single soliton: There exist $\sigma= \pm 1, \ell \in \mathbb{R}$ such that $\vec{u}(t)$ converges exponentially to $(\sigma Q(\cdot-\ell), 0)$ in $H^{1} \times L^{2}$ as $t \rightarrow \infty$.

Multi-soliton: There exist $K \geq 2, \sigma= \pm 1, \ell \in \mathbb{R}$ and functions $z_{k}:[0, \infty) \rightarrow \mathbb{R}$, for all $k=1, \ldots, K$ such that for all $t \in(0, \infty)$,

$$
\left\|u(t)-\sigma \sum_{k=1}^{K}(-1)^{k} Q\left(\cdot-z_{k}(t)\right)\right\|_{H^{1}}+\left\|\partial_{t} u(t)\right\|_{L^{2}} \lesssim t^{-1},
$$

and for any $1<\theta<\min \left(p-1, \frac{5}{4}\right), k=1, \ldots, K$,

$$
z_{k}(t)=\left(k-\frac{K+1}{2}\right) \log t+\tau_{k}+\ell+O\left(t^{-\theta+1}\right),
$$

as $t \rightarrow \infty$, where $\tau_{k}$ are the constants uniquely defined by

$$
\sum_{k=1}^{K} \tau_{k}=0, \quad e^{-\left(\tau_{k+1}-\tau_{k}\right)}=\frac{2 \alpha}{\kappa} \gamma_{k} \quad \text { where } \gamma_{k}=\frac{k(K-k)}{2},
$$

and $\kappa>0$ defined in (3.8) only depends on $p$.

Remark 1.2. The parameter $\ell$ is related to the translation invariance. In the vanishing and the single soliton cases, the damping leads to exponential convergence as $t \rightarrow \infty$. In the multi-soliton case, due to the nonlinear interactions between the solitary waves, the asymptotic behavior (1.7) of the centers of mass $z_{k}$ is related to the following nonlinear differential system

$$
\left\{\begin{array}{l}
\dot{y}_{1}=-\frac{\kappa}{2 \alpha} e^{-\left(y_{2}-y_{1}\right)}, \\
\dot{y}_{k}=\frac{\kappa}{2 \alpha}\left(e^{-\left(y_{k}-y_{k-1}\right)}-e^{-\left(y_{k+1}-y_{k}\right)}\right), \quad \text { for } k=2, \cdots, K-1, \\
\dot{y}_{K}=\frac{\kappa}{2 \alpha} e^{-\left(y_{K}-y_{K}-1\right)} .
\end{array}\right.
$$

This system is studied in [8, 16] in the context of blowup solutions of the nonlinear wave equation. The nonlinear interactions at short distances between the solitary waves also yield the slower rate of convervence $t^{-1}$ in (1.6). See [12, 17, 18, for other examples of strong interactions leading to $\log t$ distant solitary waves.

Theorem 1.1 is a version of the soliton resolution for global solutions of the onedimensional damped nonlinear Klein-Gordon equation (1.1), with convergence for the whole sequence of time and a description of the parameters of the solitary waves. The case of the damped Klein-Gordon equation on a bounded domain for suitable nonlinearities is addressed in [3. Theorem 9.5.3]; see also references therein.

We refer to [4, 9, 10, for results related to the soliton resolution conjecture for the challenging case of the undamped energy critical wave type equation.

Obviously, $\vec{u}=(0,0)$ and $\vec{u}=(Q, 0)$ are examples of the first two scenarios of Theorem 1.1. Our second result gives examples of the third scenario for any $K \geq 2$.

Theorem 1.3. For any $K \geq 2, \sigma= \pm 1$ and $\ell \in \mathbb{R}$, there exist global solutions of (1.1) satisfying (1.6) and (1.7). 
1.2. Notation and basic results on the solitary wave. We denote $\langle\cdot, \cdot\rangle$ the $L^{2}$ scalar product for real-valued functions $u_{i}$ or vector-valued functions $\vec{u}_{i}=\left(u_{i}, v_{i}\right)$ $(i=1,2)$

$$
\left\langle u_{1}, u_{2}\right\rangle:=\int u_{1}(x) u_{2}(x) \mathrm{d} x, \quad\left\langle\vec{u}_{1}, \vec{u}_{2}\right\rangle:=\int u_{1}(x) u_{2}(x) \mathrm{d} x+\int v_{1}(x) v_{2}(x) \mathrm{d} x .
$$

We see from the explicit expression of $Q$ in (1.4) that, as $x \rightarrow \infty$,

$$
Q(x)=c_{Q} e^{-x}+O\left(e^{-2 x}\right), \quad Q^{\prime}(x)=-c_{Q} e^{-x}+O\left(e^{-2 x}\right)
$$

where $c_{Q}=(2 p+2)^{\frac{1}{p-1}}$. Note that by (1.5), it holds $\int\left(\partial_{x} Q\right)^{2}+Q^{2}-Q f(Q)=0$ and so

Let

$$
E(Q, 0)=\left(\frac{1}{2}-\frac{1}{p+1}\right) \int Q^{p+1}>0 .
$$

$$
\mathcal{L}=-\partial_{x}^{2}+1-p Q^{p-1}, \quad\langle\mathcal{L} \varepsilon, \varepsilon\rangle=\int\left\{\left|\partial_{x} \varepsilon\right|^{2}+\varepsilon^{2}-p Q^{p-1} \varepsilon^{2}\right\} \mathrm{d} x .
$$

We recall some standard properties of the operator $\mathcal{L}$ (see e.g. [7, Lemma 1]).

Lemma 1.4. The following properties hold.

(i) Spectral properties. The unbounded operator $\mathcal{L}$ on $L^{2}$ with domain $H^{2}$ is self-adjoint, its continuous spectrum is $[1, \infty)$, its kernel is $\operatorname{span}\left\{Q^{\prime}\right\}$ and it has a unique negative eigenvalue $-\nu_{0}^{2}$, with corresponding smooth normalized eigenfunction $Y\left(\|Y\|_{L^{2}}=1\right)$. Moreover, on $\mathbb{R}$,

$$
\left|Y^{(n)}(x)\right| \lesssim e^{-\sqrt{1+\nu_{0}^{2}}|x|} \quad \text { for any } n \in \mathbb{N}
$$

(ii) Coercivity property. There exists $c>0$ such that, for all $\varepsilon \in H^{1}$,

$$
\langle\mathcal{L} \varepsilon, \varepsilon\rangle \geq c\|\varepsilon\|_{H^{1}}^{2}-c^{-1}\left(\langle\varepsilon, Y\rangle^{2}+\left\langle\varepsilon, Q^{\prime}\right\rangle^{2}\right) .
$$

Recall that the unique negative eigenvalue of $\mathcal{L}$ is related to an instability of the solitary wave for the equation (1.1), described by the following functions:

$$
\begin{array}{cc}
\nu^{ \pm}=-\alpha \pm \sqrt{\alpha^{2}+\nu_{0}^{2}}, \quad \vec{Y}^{ \pm}=\left(\begin{array}{c}
Y \\
\nu^{ \pm} Y
\end{array}\right), \\
\zeta^{ \pm}=\alpha \pm \sqrt{\alpha^{2}+\nu_{0}^{2}}, \quad \vec{Z}^{ \pm}=\left(\begin{array}{c}
\zeta^{ \pm} Y \\
Y
\end{array}\right) .
\end{array}
$$

\section{General Properties of Finite ENERGy SOLUtions}

In this section, we gather some known material on finite energy solutions of (1.1). We repeat some proofs for the sake of completeness.

2.1. Cauchy problem in the energy space. It is well-known (see for instance [3. Chapter 9.5]) that the linear problem

$$
\partial_{t}^{2} u+2 \alpha \partial_{t} u-\partial_{x}^{2} u+u=0 \quad(t, x) \in \mathbb{R} \times \mathbb{R}
$$

generates a strongly continuous semigroup of contractions $\left(S_{\alpha}(t)\right)_{t \geq 0}$ in $H^{1} \times L^{2}$ or $L^{2} \times H^{-1}$ satisfying, for some $C \geq 1, \gamma>0$,

$$
\left\|S_{\alpha}(t)\right\|_{\mathcal{L}\left(H^{1} \times L^{2}\right)} \leq C e^{-\gamma t}, \quad\left\|S_{\alpha}(t)\right\|_{\mathcal{L}\left(L^{2} \times H^{-1}\right)} \leq C e^{-\gamma t},
$$

for all $t \geq 0$. Recall also that the map $u \mapsto f(u)$ is Lipschitz continuous from bounded sets of $H^{1}$ to $L^{2}$. In particular, the standard theory of semilinear evolution equations (see for instance [3, Chapter 4.3] or [19]) yields the following result. 
Proposition 2.1. For any initial data $\left(u_{0}, v_{0}\right) \in H^{1} \times L^{2}$, there exists a unique maximal solution

$$
\vec{u}=\left(u, \partial_{t} u\right) \in C\left(\left[0, T_{\max }\right), H^{1} \times L^{2}\right) \cap C^{1}\left(\left[0, T_{\max }\right), L^{2} \times H^{-1}\right)
$$

of (1.1) satisfying $\vec{u}(0)=\left(u_{0}, v_{0}\right)$. If the maximal time of existence $T_{\max }$ is finite, then $\lim _{t \uparrow T_{\max }}\|\vec{u}(t)\|_{H^{1} \times L^{2}}=\infty$.

Moreover, the map $T_{\max }:\left(u_{0}, v_{0}\right) \in H^{1} \times L^{2} \mapsto(0, \infty]$ is lower semicontinuous, and if $\left(u_{0, n}, v_{0, n}\right) \rightarrow_{n \rightarrow \infty}\left(u_{0}, v_{0}\right)$ in $H^{1} \times L^{2}$ then, for any $0<T<T_{\max }$,

$$
\left(u_{n}, \partial_{t} u_{n}\right) \rightarrow\left(u, \partial_{t} u\right) \quad \text { in } C\left([0, T], H^{1} \times L^{2}\right),
$$

where $\left(u_{n}, \partial_{t} u_{n}\right)$ is the solution of (1.1) corresponding to $\left(u_{0, n}, v_{0, n}\right)$.

In this paper, we systematically work in the framework of such maximal finite energy solutions, for which it is standard to check that the relation (1.3) holds. We call global solution a solution for which $T_{\max }=\infty$. We do not consider solutions of (1.1) backwards in time (i.e. for negative values of $t$ ).

2.2. Bound on global solutions. Gathering the arguments of [2] and 11, Proof of Lemma 2.7], we recall the following bound on global solutions of (1.1). Note that [2] is devoted to the undamped Klein-Gordon equation, but as suggested in the Introduction of [1, the proof extends to the damped case.

Theorem 2.2 ([1, 2]). Any global solution of (1.1) is bounded in $H^{1} \times L^{2}$.

Proof. Let $\vec{u}$ be a global solution of (1.1). Together with the energy functional $E(t):=E(\vec{u}(t))$ defined in (1.2) and satisfying (1.3), we will use the following quantities

$$
\begin{aligned}
M(t) & :=\frac{1}{2}\|u(t)\|_{L^{2}}^{2}+\alpha \int_{0}^{t}\|u(s)\|_{L^{2}}^{2} \mathrm{~d} s, \\
W(t) & :=\frac{1}{2}\left(\left\|\partial_{t} u(t)\right\|_{L^{2}}^{2}+\left\|\partial_{x} u(t)\right\|_{L^{2}}^{2}+\|u(t)\|_{L^{2}}^{2}\right) .
\end{aligned}
$$

By direct computations using (1.1) and (1.2), we check the following relations

$$
\begin{aligned}
M^{\prime}(t) & =\int u(t) \partial_{t} u(t) \mathrm{d} x+\alpha\|u(t)\|_{L^{2}}^{2} \\
& =\int u(t) \partial_{t} u(t) \mathrm{d} x+2 \alpha \int_{0}^{t} \int u(s) \partial_{t} u(s) \mathrm{d} x \mathrm{~d} s+\alpha\|u(0)\|_{L^{2}}^{2}, \\
M^{\prime \prime}(t) & =\frac{p+3}{2}\left\|\partial_{t} u(t)\right\|_{L^{2}}^{2}+\frac{p-1}{2}\left(\left\|\partial_{x} u(t)\right\|_{L^{2}}^{2}+\|u(t)\|_{L^{2}}^{2}\right)-(p+1) E(t), \\
W^{\prime}(t) & =-2 \alpha\left\|\partial_{t} u(t)\right\|_{L^{2}}^{2}+\int f(u(t)) \partial_{t} u(t) \mathrm{d} x .
\end{aligned}
$$

In particular, from (2.2) and the Cauchy-Schwarz inequality,

$$
\left|M^{\prime}(t)\right| \leq(1+2 \alpha) W(t) .
$$

Moreover, by (1.3) and (2.4),

$$
M^{\prime \prime}(t) \geq(p-1) W(t)-(p+1) E(0) .
$$

The proof of the global bound now proceeds in three steps.

Step 1. We prove that

$$
\liminf _{t \rightarrow \infty} M^{\prime}(t)<\infty
$$

Proof of (2.8). We argue by contradiction, proving that $\lim _{\infty} M^{\prime}=\infty$ implies the following inequality, for all $t$ large enough,

$$
(1+\epsilon)\left[M^{\prime}(t)\right]^{2}<M^{\prime \prime}(t) M(t) \quad \text { where } \quad \epsilon>0 .
$$


Then, we reach a contradiction by a standard argument. Indeed, remark that (2.9) implies $\frac{\mathrm{d}^{2}}{\mathrm{~d} t^{2}}\left[M^{-\epsilon}(t)\right]<0$, and $\lim _{\infty} M^{\prime}=\infty$ also implies $\lim _{\infty} M^{-\epsilon}=0$. Thus, there exists $t_{1}>0$ such that $\frac{\mathrm{d}}{\mathrm{d} t}\left[M^{-\epsilon}\left(t_{1}\right)\right]<0$, and for all $t \geq t_{1}$,

$$
0 \leq M^{-\epsilon}(t) \leq M^{-\epsilon}\left(t_{1}\right)+\left(t-t_{1}\right) \frac{\mathrm{d}}{\mathrm{d} t}\left[M^{-\epsilon}\left(t_{1}\right)\right]
$$

which is absurd for $t \geq t_{1}$ large enough.

Thus, we only need to prove (2.9) assuming $\lim _{\infty} M^{\prime}=\infty$. On the one hand, by (2.3) and the Cauchy-Schwarz inequality, it holds

$\left|M^{\prime}\right| \leq\|u\|_{L^{2}}\left\|\partial_{t} u\right\|_{L^{2}}+2 \alpha\left(\int_{0}^{t}\|u(s)\|_{L^{2}}^{2} \mathrm{~d} s\right)^{\frac{1}{2}}\left(\int_{0}^{t}\left\|\partial_{t} u(s)\right\|_{L^{2}}^{2} \mathrm{~d} s\right)^{\frac{1}{2}}+\alpha\|u(0)\|_{L^{2}}^{2}$.

Let $\epsilon>0$ to be chosen later, we estimate

$$
\begin{aligned}
\left|M^{\prime}\right|^{2} \leq & (1+\epsilon)\left[\|u\|_{L^{2}}\left\|\partial_{t} u\right\|_{L^{2}}+2 \alpha\left(\int_{0}^{t}\|u(s)\|_{L^{2}}^{2} \mathrm{~d} s\right)^{\frac{1}{2}}\left(\int_{0}^{t}\left\|\partial_{t} u(s)\right\|_{L^{2}}^{2} \mathrm{~d} s\right)^{\frac{1}{2}}\right]^{2} \\
& +\left(1+\frac{1}{\epsilon}\right) \alpha^{2}\|u(0)\|_{L^{2}}^{4}
\end{aligned}
$$

Thus,

$$
\begin{aligned}
\left|M^{\prime}\right|^{2} \leq & (1+\epsilon)\left[\frac{1}{2}\|u\|_{L^{2}}^{2}+\alpha \int_{0}^{t}\|u(s)\|_{L^{2}}^{2} \mathrm{~d} s\right]\left[2\left\|\partial_{t} u\right\|_{L^{2}}^{2}+4 \alpha \int_{0}^{t}\left\|\partial_{t} u(s)\right\|_{L^{2}}^{2} \mathrm{~d} s\right] \\
& +\left(1+\frac{1}{\epsilon}\right) \alpha^{2}\|u(0)\|_{L^{2}}^{4} \\
\leq & (1+\epsilon) M\left[2\left\|\partial_{t} u\right\|_{L^{2}}^{2}+4 \alpha \int_{0}^{t}\left\|\partial_{t} u(s)\right\|_{L^{2}}^{2} \mathrm{~d} s\right]+\left(1+\frac{1}{\epsilon}\right) \alpha^{2}\|u(0)\|_{L^{2}}^{4} .
\end{aligned}
$$

On the other hand, by (1.3) and (2.4),

$$
\begin{aligned}
M^{\prime \prime} & =2\left\|\partial_{t} u\right\|_{L^{2}}^{2}+(p-1) W+2 \alpha(p+1) \int_{0}^{t}\left\|\partial_{t} u(s)\right\|_{L^{2}}^{2} \mathrm{~d} s-(p+1) E(0) \\
& \geq(1+\epsilon)^{3}\left[2\left\|\partial_{t} u\right\|_{L^{2}}^{2}+4 \alpha \int_{0}^{t}\left\|\partial_{t} u(s)\right\|_{L^{2}}^{2} \mathrm{~d} s\right]+\frac{p-1}{2} W(t)-(p+1) E(0),
\end{aligned}
$$

by fixing any $\epsilon$ such that

$$
0<\epsilon<\left(\frac{p+7}{8}\right)^{\frac{1}{3}}-1
$$

In particular, since $\lim _{\infty} W=\infty$ by (2.6) and the assumption $\lim _{\infty} M^{\prime}=\infty$, we have for $t$ large enough,

$$
M^{\prime \prime} \geq(1+\epsilon)^{3}\left[2\left\|\partial_{t} u\right\|_{L^{2}}^{2}+4 \alpha \int_{0}^{t}\left\|\partial_{t} u(s)\right\|^{2} \mathrm{~d} s\right]
$$

Thus,

$$
(1+\epsilon)^{2}\left|M^{\prime}\right|^{2} \leq M M^{\prime \prime}+\left(1+\frac{1}{\epsilon}\right) \alpha^{2}\|u(0)\|_{L^{2}}^{4}
$$

and using again $\lim _{\infty} M^{\prime}=\infty$ we obtain (2.9) for any $t$ large enough.

Step 2. We prove that

$$
\sup _{t \in[0, \infty)}\left|M^{\prime}(t)\right|<\infty .
$$

Proof of (2.10). Combining (2.6) and (2.7), we obtain

$$
M^{\prime \prime}(t) \geq \frac{p-1}{1+2 \alpha}\left|M^{\prime}(t)\right|-(p+1) E(0) .
$$


Let

$$
H(t)=\frac{p-1}{1+2 \alpha} M^{\prime}(t)-(p+1) E(0) .
$$

Then, $H^{\prime}(t)=\frac{p-1}{1+2 \alpha} M^{\prime \prime}(t) \geq \frac{p-1}{1+2 \alpha} H(t)$. If there exists $t \geq 0$ such that $H(t)>0$, then $\lim _{\infty} H=\infty$, contradicting (2.8). It follows that for all $t \geq 0$,

$$
M^{\prime}(t) \leq \frac{1+2 \alpha}{p-1}(p+1) E(0)
$$

Similarly, let

$$
K(t)=-\frac{p-1}{1+2 \alpha} M^{\prime}(t)+(p+1) E(0) .
$$

Then, $K^{\prime}(t)=-\frac{p-1}{1+2 \alpha} M^{\prime \prime}(t) \leq-\frac{p-1}{1+2 \alpha} K(t)$. It follows that $K(t) \leq e^{-\frac{p-1}{1+2 \alpha} t} K(0)$, for all $t \geq 0$. Thus,

$$
M^{\prime}(t) \geq-\frac{1+2 \alpha}{p-1}((p+1) E(0)+|K(0)|) .
$$

and (2.10) is proved.

Step 3. Last, we prove the global bound

$$
\sup _{t \in[0, \infty)}|W(t)|<\infty .
$$

Proof of (2.11). We rewrite (2.7) as

$$
W(t) \leq \frac{1}{p-1} M^{\prime \prime}(t)+\frac{p+1}{p-1} E(0) .
$$

Integrating on $(t, t+1)$ and using (2.10), we observe that

$$
\sup _{t \geq 0} \int_{t}^{t+1} W(s) \mathrm{d} s<\infty .
$$

Moreover, by (2.5),

$$
W^{\prime} \leq-2 \alpha\left\|\partial_{t} u\right\|_{L^{2}}^{2}+\int|u|^{p}\left|\partial_{t} u\right| \leq \frac{1}{2}\left\|\partial_{t} u\right\|_{L^{2}}^{2}+\frac{1}{2} \int|u|^{2 p} \leq W+\frac{1}{2} \int|u|^{2 p} .
$$

For $t \geq 1$ and $\tau \in(0,1)$, integrating on $(t-\tau, t)$, we find

$$
\begin{aligned}
W(t) & \leq W(t-\tau)+\int_{t-\tau}^{t} W(s) \mathrm{d} s+\frac{1}{2} \int_{t-\tau}^{t} \int|u(s)|^{2 p} \mathrm{~d} x \mathrm{~d} s \\
& \leq W(t-\tau)+\int_{t-1}^{t} W(s) \mathrm{d} s+\frac{1}{2} \int_{t-1}^{t} \int|u(s)|^{2 p} \mathrm{~d} x \mathrm{~d} s .
\end{aligned}
$$

Using the Sobolev inequality (in space-time) for the last term, we obtain, for some constants $C>0$,

$$
\begin{aligned}
W(t) & \leq W(t-\tau)+\int_{t-1}^{t} W(s) \mathrm{d} s+C\|u\|_{H^{1}((t-1, t) \times \mathbb{R})}^{2 p} \\
& \leq W(t-\tau)+\int_{t-1}^{t} W(s) \mathrm{d} s+C\left(\int_{t-1}^{t} W(s) \mathrm{d} s\right)^{p} .
\end{aligned}
$$

Integrating in $\tau \in(0,1)$ and using (2.12), we find (2.11). 


\subsection{Decomposition of any global solution along a subsequence.}

Theorem 2.3 (11, 13, 14]). Any global solution $\vec{u}$ of (1.1)

- either converges to 0 , i.e. $\lim _{t \rightarrow \infty}\|\vec{u}(t)\|_{H^{1} \times L^{2}}=0$;

- or is asymptotically a (multi-)solitary wave along a subsequence of time: there exist $K \geq 1$, a sequence $t_{n} \rightarrow \infty$, a sequence $\left(\xi_{k, n}\right)_{k \in\{1, \ldots, K\}} \in \mathbb{R}^{K}$ and signs $\sigma_{k}= \pm 1$, for any $k \in\{1, \ldots, K\}$, such that

$$
\lim _{n \rightarrow \infty}\left\{\left\|u\left(t_{n}\right)-\sum_{k=1}^{K} \sigma_{k} Q\left(\cdot-\xi_{k, n}\right)\right\|_{H^{1}}+\left\|\partial_{t} u\left(t_{n}\right)\right\|_{L^{2}}\right\}=0
$$

and in the case $K \geq 2$,

$$
\lim _{n \rightarrow \infty} \xi_{k+1, n}-\xi_{k, n}=\infty \quad \text { for any } 1 \leq k \leq K-1
$$

Remark 2.4. It is clear that if a global solution $\vec{u}$ satisfies (2.13) for two different sequences $\left(t_{n}\right)_{n}$ and $\left(t_{n}^{\prime}\right)_{n}$, then the number $K \geq 1$ of solitary waves is the same for both sequences. Indeed, by monotonicity of the energy (1.3) and (1.11), it holds

$$
\lim _{t \rightarrow \infty} E(\vec{u}(t))=K E(Q, 0)>0 \text {. }
$$

Remark 2.5. The following stronger result holds in the framework of Theorem 2.3 . for any sequence $\left(t_{n}\right)_{n}$ with $t_{n} \rightarrow \infty$, the multi-solitary wave behavior (2.13) is satisfied for a subsequence of $\left(t_{n}\right)_{n}$. This result, valid on any global solution of (1.1), is quite remarkable. However, it does not fully describe the asymptotic behavior of global solutions as $t \rightarrow \infty$, which is the objective of Theorem 1.1 .

Remark 2.6. Note that [1, 2, 6, 11, 13, 14, 15, also apply to the multi-dimensional case, under suitable restrictions on the exponent $p$ of the nonlinearity, or for radially symmetric solutions. However, for space dimensions greater than 1 , the existence of bound states solutions of $\Delta w-w+f(w)=0$ other than the ground state $Q$, together with the possibility of involved geometric configurations of solitary waves, complicate the analysis. This is why we restrict to dimension 1 in the present paper.

Proof. Let $\vec{u}$ be a global solution of (1.1); in particular, by Theorem 2.2. it is bounded in $H^{1} \times L^{2}$. The proof proceeds in two steps.

Step 1. We prove that

$$
\lim _{t \rightarrow \infty}\left\{\left\|\partial_{t} u(t)\right\|_{L^{2}}+\left\|\partial_{t}^{2} u(t)\right\|_{H^{-1}}\right\}=0 .
$$

With the notation of 2.1 the function $\vec{v}(t)=\left(v(t), \partial_{t} v(t)\right)=\left(\partial_{t} u(t), \partial_{t}^{2} u(t)\right)$ satisfies

$$
\vec{v}(t)=S_{\alpha}(t) \vec{v}(0)+\int_{0}^{t} S_{\alpha}(t-s)\left(0, p|u(s)|^{p-1} v(s)\right) \mathrm{d} s .
$$

By the bound in $H^{1} \times L^{2}$ and (1.3) , it follows that $v \in L^{2}((0, \infty) \times \mathbb{R})$. Moreover, using estimate (2.1), $\|\cdot\|_{H^{-1}} \lesssim\|\cdot\|_{L^{2}}$ and $\|\cdot\|_{L^{\infty}} \lesssim\|\cdot\|_{H^{1}}$, we have, for all $t \geq 0$,

$$
\|\vec{v}(t)\|_{L^{2} \times H^{-1}} \lesssim e^{-\gamma t}\|\vec{v}(0)\|_{L^{2} \times H^{-1}}+\|u\|_{L^{\infty}\left([0, \infty), H^{1}\right)}^{p-1} \int_{0}^{t} e^{-\gamma(t-s)}\|v(s)\|_{L^{2}} \mathrm{~d} s .
$$

Splitting the integral $\int_{0}^{t}=\int_{0}^{t / 2}+\int_{t / 2}^{t}$ in the last term and using the CauchySchwarz inequality

$$
\int_{0}^{t} e^{-\gamma(t-s)}\|v(s)\|_{L^{2}} \mathrm{~d} s \lesssim e^{-\gamma t / 2}\|v\|_{L^{2}((0, \infty) \times \mathbb{R})}+\|v\|_{L^{2}((t / 2, \infty) \times \mathbb{R})},
$$

which implies $\lim _{t \rightarrow \infty}\|\vec{v}(t)\|_{L^{2} \times H^{-1}}=0$ and thus (2.15). 
Step 2. Let $\left(t_{n}\right)_{n}$ be any sequence such that $t_{n} \rightarrow \infty$ and let $u_{n}(x)=u\left(t_{n}, x\right)$. Then, by (2.15) and equation (1.1), it follows that

$$
\lim _{n \rightarrow \infty}\left\|\partial_{x}^{2} u_{n}-u_{n}+\left|u_{n}\right|^{p-1} u_{n}\right\|_{H^{-1}}=0 .
$$

Moreover, the sequence $\left(u_{n}\right)_{n}$ is bounded in $H^{1}$. Then, the alternative stated in the Theorem follows directly from results from concentration-compactness arguments in [13. Appendix A] and [14, Theorem III.4]. In the present framework, we use [14, Theorem III.4] in space dimension 1 and with the constant coefficient elliptic operator $-\partial_{x}^{2}+1$, which simplifies the statement. Observe that in dimension 1 , we enjoy the fact that the only non trivial solutions of $\partial_{x}^{2} w-w+f(w)=0$ are $w= \pm Q$, up to space translation.

In the case where $\lim _{n \rightarrow \infty}\left\|\vec{u}\left(s_{n}\right)\right\|_{H^{1} \times L^{2}}=0$, for some sequence of time $\left(s_{n}\right)_{n}$, $s_{n} \rightarrow \infty$, then it follows from (1.3) that $\lim _{t \rightarrow \infty} E(\vec{u}(t))=0$. Thus, by (1.11) and the previous arguments applied to any sequence $\left(t_{n}\right)_{n}$, with $t_{n} \rightarrow \infty$, there exists a subsequence $\left(t_{n^{\prime}}\right)_{n^{\prime}}$ such that $\lim _{n^{\prime} \rightarrow \infty}\left\|\vec{u}\left(t_{n^{\prime}}\right)\right\|_{H^{1} \times L^{2}}=0$. This implies that $\lim _{t \rightarrow \infty}\|\vec{u}(t)\|_{H^{1} \times L^{2}}=0$ as stated in the first part of the alternative.

\section{Dynamics Close to DeCoupled solitary WAVES}

In this Section, we prove general results on solutions of (1.1) close to the sum of $K \geq 1$ decoupled solitary waves. For any $k \in\{1, \cdots, K\}$, let $\sigma_{k}= \pm 1$ and let $t \mapsto\left(z_{k}(t), \ell_{k}(t)\right) \in \mathbb{R}^{2}$ be $\mathcal{C}^{1}$ functions such that

$$
\sum_{k=1}^{K}\left|\ell_{k}\right| \ll 1 \quad \text { and, if } K \geq 2 \text {, for any } k=1, \ldots, K-1, \quad z_{k+1}-z_{k} \gg 1 \text {. }
$$

For $k \in\{1, \cdots, K\}$, define

$$
Q_{k}=\sigma_{k} Q\left(\cdot-z_{k}\right), \quad \vec{Q}_{k}=\left(\begin{array}{c}
Q_{k} \\
-\ell_{k} \partial_{x} Q_{k}
\end{array}\right),
$$

and similarly (see (1.12)-(1.13)

$$
Y_{k}=\sigma_{k} Y\left(\cdot-z_{k}\right), \quad \vec{Y}_{k}^{ \pm}=\sigma_{k} \vec{Y}^{ \pm}\left(\cdot-z_{k}\right), \quad \vec{Z}_{k}^{ \pm}=\sigma_{k} \vec{Z}^{ \pm}\left(\cdot-z_{k}\right) .
$$

Set

$$
R=\sum_{k=1}^{K} Q_{k}, \quad \vec{R}=\sum_{k=1}^{K} \vec{Q}_{k}, \quad G=f\left(\sum_{k=1}^{K} Q_{k}\right)-\sum_{k=1}^{K} f\left(Q_{k}\right) .
$$

\subsection{Leading order of the nonlinear interactions.}

Lemma 3.1. Assuming (3.1), for any $k, k^{\prime} \in\{1, \ldots, K\}, k^{\prime} \neq k$, it holds.

(i) Bounds. For any $0<m^{\prime}<m$,

$$
\begin{gathered}
\int\left|Q_{k^{\prime}} Q_{k}\right|^{m} \lesssim e^{-m^{\prime}\left|z_{k^{\prime}}-z_{k}\right|}, \quad \int\left|Q_{k^{\prime}}\right|\left|Q_{k}\right|^{1+m} \lesssim e^{-\left|z_{k^{\prime}}-z_{k}\right|}, \\
\int\left|F(R)-\sum_{k=1}^{K} F\left(Q_{k}\right)-\sum_{k \neq k^{\prime}} f\left(Q_{k}\right) Q_{k^{\prime}}\right| \lesssim \sum_{k=1}^{K-1} e^{-\frac{5}{4}\left(z_{k+1}-z_{k}\right)}, \\
\|G\|_{L^{2}} \lesssim \sum_{k^{\prime} \neq k}\left\|Q_{k^{\prime}}^{p-1} Q_{k}\right\|_{L^{2}} \lesssim \sum_{k=1}^{K-1} e^{-\left(z_{k+1}-z_{k}\right)} .
\end{gathered}
$$

(ii) Asymptotics.

$$
\left|\left\langle f\left(Q_{k}\right), Q_{k^{\prime}}\right\rangle-\sigma_{k} \sigma_{k^{\prime}} c_{1} \kappa e^{-\left|z_{k}-z_{k^{\prime}}\right|}\right| \lesssim e^{-\frac{3}{2}\left|z_{k}-z_{k^{\prime}}\right|}
$$


where

$$
\kappa:=\frac{c_{Q}}{c_{1}} \int Q^{p}(x) e^{-x} \mathrm{~d} x>0 \quad \text { and } \quad c_{1}:=\left\|Q^{\prime}\right\|_{L^{2}}^{2} .
$$

(iii) Leading order interactions. Let any $1<\theta<\min \left(p-1, \frac{3}{2}\right)$.

- If $K=1$ then $G=0$;

- If $K \geq 2$ then

$$
\begin{gathered}
\left|\left\langle G, \partial_{x} Q_{1}\right\rangle+c_{1} \kappa \sigma_{1} \sigma_{2} e^{-\left(z_{2}-z_{1}\right)}\right| \lesssim \sum_{l=1}^{K-1} e^{-\theta\left(z_{l+1}-z_{l}\right)} \\
\left|\left\langle G, \partial_{x} Q_{K}\right\rangle-c_{1} \kappa \sigma_{K-1} \sigma_{K} e^{-\left(z_{K}-z_{K-1}\right)}\right| \lesssim \sum_{l=1}^{K-1} e^{-\theta\left(z_{l+1}-z_{l}\right)} ;
\end{gathered}
$$

- If $K \geq 3$ then, for any $k \in\{2, \ldots, K-1\}$,

$$
\begin{aligned}
& \left|\left\langle G, \partial_{x} Q_{k}\right\rangle-c_{1} \kappa \sigma_{k}\left[\sigma_{k-1} e^{-\left(z_{k}-z_{k-1}\right)}-\sigma_{k+1} e^{-\left(z_{k+1}-z_{k}\right)}\right]\right| \\
& \quad \lesssim \sum_{l=1}^{K-1} e^{-\theta\left(z_{l+1}-z_{l}\right)}
\end{aligned}
$$

Proof. Proof of (i). These estimates are direct consequences of the decay properties of $Q$ in (1.10) and $p>2$. See details in [6, proof of Lemma 2.1].

Proof of (ii). We claim the following estimate for $z \gg 1$.

$$
\left|\int Q^{p}(y) Q(y+z) \mathrm{d} y-c_{1} \kappa e^{-z}\right| \lesssim e^{-\frac{3}{2} z} .
$$

Observe that (3.7) follows directly from (3.12).

Now, we prove (3.12). First, for $|y|<\frac{3}{4} z$, using (1.10), we have

$$
\left|Q(y+z)-c_{Q} e^{-(y+z)}\right| \lesssim e^{-2(y+z)} \lesssim e^{-2 z} e^{2|y|},
$$

and so

$$
\left|\int_{|y|<\frac{3}{4} z} Q^{p}(y)\left[Q(y+z)-c_{Q} e^{-(y+z)}\right] \mathrm{d} y\right| \lesssim e^{-2 z} .
$$

Second, using (1.10) and $p>2$, it holds

$$
\int_{|y|>\frac{3}{4} z} Q^{p}(y) Q(y+z) \mathrm{d} y+\int_{|y|>\frac{3}{4} z} Q^{p}(y) e^{-(y+z)} \mathrm{d} y \lesssim e^{-\frac{3}{2} z} .
$$

Gathering these estimates, we have proved (3.12).

Proof of (iii). We treat the case $k \in\{2, \ldots, K-1\}$ for $K \geq 3$. Other cases are similar. On the one hand, using Taylor formula, it holds

$$
\left.\left.|G-p| Q_{k}\right|^{p-1} \sum_{k^{\prime} \neq k} Q_{k^{\prime}}|\lesssim| Q_{k}\right|^{p-2} \sum_{k^{\prime} \neq k}\left|Q_{k^{\prime}}\right|^{2}+\sum_{k^{\prime} \neq k, l \neq k^{\prime}}\left|Q_{k^{\prime}}\right|^{p-1}\left|Q_{l}\right| .
$$

Thus, using (3.4), we have for any $1<\theta<\min (p-1,2)$,

$$
\begin{aligned}
\left|\left\langle G, \partial_{x} Q_{k}\right\rangle-\sum_{k^{\prime} \neq k}\left\langle p\left|Q_{k}\right|^{p-1} Q_{k^{\prime}}, \partial_{x} Q_{k}\right\rangle\right| & \lesssim \sum_{l \neq k^{\prime}} \int\left|Q_{k^{\prime}}\right|^{p-1}\left|Q_{l}\right|^{2} \mathrm{~d} x \\
& \lesssim \sum_{l=1}^{K-1} e^{-\theta\left(z_{l+1}-z_{l}\right)} .
\end{aligned}
$$


On the other hand, by direct computation, integrating by parts and using the proof of (3.12), we obtain, for any $1<\theta<\min \left(p-1, \frac{3}{2}\right)$,

$$
\begin{aligned}
& \sum_{k^{\prime} \neq k}\left\langle p\left|Q_{k}\right|^{p-1} Q_{k^{\prime}}, \partial_{x} Q_{k}\right\rangle \\
& =-\sum_{k^{\prime} \neq k} \sigma_{k} \sigma_{k^{\prime}} \int Q^{p}(y) \partial_{x} Q\left(y+z_{k}-z_{k^{\prime}}\right) \mathrm{d} y \\
& =\sum_{k^{\prime}<k} \sigma_{k^{\prime}} \sigma_{k} c_{1} \kappa e^{-\left(z_{k}-z_{k^{\prime}}\right)}-\sum_{k^{\prime}>k} \sigma_{k} \sigma_{k^{\prime}} c_{1} \kappa e^{-\left(z_{k^{\prime}}-z_{k}\right)}+O\left(\sum_{l=1}^{K-1} e^{-\frac{3}{2}\left(z_{l+1}-z_{l}\right)}\right) \\
& =\sigma_{k-1} \sigma_{k} c_{1} \kappa e^{-\left(z_{k}-z_{k-1}\right)}-\sigma_{k} \sigma_{k+1} c_{1} \kappa e^{-\left(z_{k+1}-z_{k}\right)}+O\left(\sum_{l=1}^{K-1} e^{-\frac{3}{2}\left(z_{l+1}-z_{l}\right)}\right) .
\end{aligned}
$$

Indeed, for example, if $k \geq 3$, one has

$$
z_{k}-z_{k-2}=\left(z_{k}-z_{k-1}\right)+\left(z_{k-1}-z_{k-2}\right) \geq 2 \min \left(z_{k}-z_{k-1} ; z_{k-1}-z_{k-2}\right),
$$

which allows us to consider interactions between to non neighbor solitary waves as error terms. Gathering the above estimates, we find (3.11).

\subsection{Decomposition close to the sum of solitary waves.}

Lemma 3.2. Let $\vec{u}=\left(u, \partial_{t} u\right)$ be a solution of (1.1) on the interval $\left[T_{1}, T_{2}\right]$ and let $K \geq 1$. Assume that

$$
\sup _{t \in\left[T_{1}, T_{2}\right]}\left\{\inf _{\xi_{k+1}-\xi_{k}>|\log \gamma|}\left\|u(t)-\sum_{k=1}^{K} \sigma_{k} Q\left(\cdot-\xi_{k}\right)\right\|_{H^{1}}+\left\|\partial_{t} u(t)\right\|_{L^{2}}\right\}<\gamma,
$$

for some small $\gamma>0$. Then, there exist unique $\mathcal{C}^{1}$ functions

$$
t \in\left[T_{1}, T_{2}\right] \mapsto\left(z_{k}(t), \ell_{k}(t)\right)_{k \in\{1, \ldots, K\}} \in \mathbb{R}^{2 K},
$$

such that the solution $\vec{u}$ decomposes as

$$
\vec{u}=\left(\begin{array}{c}
u \\
\partial_{t} u
\end{array}\right)=\sum_{k=1}^{K} \vec{Q}_{k}+\vec{\varepsilon}, \quad \vec{\varepsilon}=\left(\begin{array}{c}
\varepsilon \\
\eta
\end{array}\right)
$$

with the following properties on $\left[T_{1}, T_{2}\right]$.

(i) Orthogonality and smallness. For any $k=1, \ldots, K$,

$$
\left\langle\varepsilon, \partial_{x} Q_{k}\right\rangle=\left\langle\eta, \partial_{x} Q_{k}\right\rangle=0
$$

and

$$
\|\vec{\varepsilon}\|_{H^{1} \times L^{2}}+\sum_{l=1}^{K}\left|\ell_{l}\right|+\sum_{l=1}^{K-1} e^{-2\left(z_{l+1}-z_{l}\right)} \lesssim \gamma .
$$

(ii) Equation of $\vec{\varepsilon}$.

$$
\left\{\begin{array}{l}
\partial_{t} \varepsilon=\eta+\operatorname{Mod}_{\varepsilon} \\
\partial_{t} \eta=\partial_{x}^{2} \varepsilon-\varepsilon+f(R+\varepsilon)-f(R)-2 \alpha \eta+\operatorname{Mod}_{\eta}+G
\end{array}\right.
$$

where

$$
\begin{aligned}
\operatorname{Mod}_{\varepsilon} & =\sum_{k=1}^{K}\left(\dot{z}_{k}-\ell_{k}\right) \partial_{x} Q_{k} \\
\operatorname{Mod}_{\eta} & =\sum_{k=1}^{K}\left(\dot{\ell}_{k}+2 \alpha \ell_{k}\right) \partial_{x} Q_{k}-\sum_{k=1}^{K} \ell_{k} \dot{z}_{k} \partial_{x}^{2} Q_{k}
\end{aligned}
$$


(iii) Control of the geometric parameters. For $k=1, \ldots, K$,

$$
\begin{aligned}
\left|\dot{z}_{k}-\ell_{k}\right| & \lesssim\|\vec{\varepsilon}\|_{H^{1} \times L^{2}}^{2}+\sum_{l=1}^{K}\left|\ell_{l}\right|^{2} \\
\left|\dot{\ell}_{k}+2 \alpha \ell_{k}\right| & \lesssim\|\vec{\varepsilon}\|_{H^{1} \times L^{2}}^{2}+\sum_{l=1}^{K}\left|\ell_{l}\right|^{2}+\sum_{l=1}^{K-1} e^{-\left(z_{l+1}-z_{l}\right)} .
\end{aligned}
$$

(iv) Control of the exponential directions. For $k=1, \cdots, K$, let

$$
a_{k}^{ \pm}=\left\langle\vec{\varepsilon}, \vec{Z}_{k}^{ \pm}\right\rangle
$$

Then,

$$
\left|\frac{\mathrm{d}}{\mathrm{d} t} a_{k}^{ \pm}-\nu^{ \pm} a_{k}^{ \pm}\right| \lesssim\|\vec{\varepsilon}\|_{H^{1} \times L^{2}}^{2}+\sum_{l=1}^{K}\left|\ell_{l}\right|^{2}+\sum_{l=1}^{K-1} e^{-\left(z_{l+1}-z_{l}\right)} .
$$

Remark 3.3. In the above estimates, if $K=1$ then the terms $\sum_{l=1}^{K-1}$ are 0 .

Proof. See [6, proof of Lemma 2.2].

Lemma 3.4 (Refined equation for $\ell_{k}$ ). In the context of Lemma 3.2, assume that $K \geq 2$. Let $1<\theta<\min \left(p-1, \frac{3}{2}\right)$. Then,

$$
\begin{aligned}
\left|\dot{\ell}_{1}+2 \alpha \ell_{1}-\kappa \sigma_{1} \sigma_{2} e^{-\left(z_{2}-z_{1}\right)}\right| & +\left|\dot{\ell}_{K}+2 \alpha \ell_{K}+\kappa \sigma_{K-1} \sigma_{K} e^{-\left(z_{K}-z_{K-1}\right)}\right| \\
& \lesssim\|\vec{\varepsilon}\|_{H^{1} \times L^{2}}^{2}+\sum_{l=1}^{K}\left|\ell_{l}\right|^{2}+\sum_{l=1}^{K-1} e^{-\theta\left(z_{l+1}-z_{l}\right)} .
\end{aligned}
$$

Moreover, if $K \geq 3$ then for any $k \in\{2, \ldots, K-1\}$,

$$
\begin{array}{r}
\left|\dot{\ell}_{k}+2 \alpha \ell_{k}+\kappa \sigma_{k}\left[\sigma_{k-1} e^{-\left(z_{k}-z_{k-1}\right)}-\sigma_{k+1} e^{-\left(z_{k+1}-z_{k}\right)}\right]\right| \\
\lesssim\|\vec{\varepsilon}\|_{H^{1} \times L^{2}}^{2}+\sum_{l=1}^{K}\left|\ell_{l}\right|^{2}+\sum_{l=1}^{K-1} e^{-\theta\left(z_{l+1}-z_{l}\right)} .
\end{array}
$$

Proof. We treat the case $k \in\{2, \ldots, K-1\}$ for $K \geq 3$. Other cases are similar. First,

$$
\frac{\mathrm{d}}{\mathrm{d} t}\left\langle\eta, \partial_{x} Q_{k}\right\rangle=\left\langle\partial_{t} \eta, \partial_{x} Q_{k}\right\rangle+\left\langle\eta, \partial_{t} \partial_{x} Q_{k}\right\rangle=0
$$

Thus, using (3.16) and (3.17),

$$
\begin{aligned}
0= & \left\langle\partial_{x}^{2} \varepsilon-\varepsilon+f^{\prime}\left(Q_{k}\right) \varepsilon, \partial_{x} Q_{k}\right\rangle+\left\langle f(R+\varepsilon)-f(R)-f^{\prime}(R) \varepsilon, \partial_{x} Q_{k}\right\rangle \\
& +\left\langle\left(f^{\prime}(R)-f^{\prime}\left(Q_{k}\right)\right) \varepsilon, \partial_{x} Q_{k}\right\rangle+\left\langle G, \partial_{x} Q_{k}\right\rangle+\left\langle\operatorname{Mod}_{\eta}, \partial_{x} Q_{k}\right\rangle-\left\langle\eta, \dot{z}_{k} \partial_{x}^{2} Q_{k}\right\rangle
\end{aligned}
$$

Since $\partial_{x} Q_{k}$ satisfies $\partial_{x}^{2} \partial_{x} Q_{k}-\partial_{x} Q_{k}+f^{\prime}\left(Q_{k}\right) \partial_{x} Q_{k}=0$, by integration by parts, the first term is zero. Next, by Taylor expansion (using $p>2$ ), we have the pointwise estimate

$$
\left|f(R+\varepsilon)-f(R)-f^{\prime}(R) \varepsilon\right| \lesssim|\varepsilon|^{p}+|\varepsilon|^{2} \sum_{l=1}^{K}\left|Q_{l}\right|^{p-2}
$$

and

$$
\left|f^{\prime}(R)-f^{\prime}\left(Q_{k}\right)\right| \lesssim\left|Q_{k}\right|^{p-2} \sum_{k^{\prime} \neq k}\left|Q_{k^{\prime}}\right|+\sum_{k^{\prime} \neq k}\left|Q_{k^{\prime}}\right|^{p-1} .
$$

Thus, using $\|\cdot\|_{L^{\infty}} \lesssim\|\cdot\|_{H^{1}}$,

$$
\left|\left\langle f(R+\varepsilon)-f(R)-f^{\prime}(R) \varepsilon, \partial_{x} Q_{k}\right\rangle\right| \lesssim\|\varepsilon\|_{H^{1}}^{2}
$$


and by the Cauchy-Schwarz inequality and (3.6),

$$
\left|\left\langle\left(f^{\prime}(R)-f^{\prime}\left(Q_{k}\right)\right) \varepsilon, \partial_{x} Q_{k}\right\rangle\right| \lesssim\|\varepsilon\|_{H^{1}}^{2}+\sum_{k=1}^{K-1} e^{-2\left(z_{k+1}-z_{k}\right)} .
$$

By direct computation, we obtain

$$
\begin{aligned}
\left\langle\operatorname{Mod}_{\eta}, \partial_{x} Q_{k}\right\rangle= & \left(\dot{\ell}_{k}+2 \alpha \ell_{k}\right)\left\|Q^{\prime}\right\|_{L^{2}}^{2}+\sum_{k^{\prime} \neq k}\left(\dot{\ell}_{k^{\prime}}+2 \alpha \ell_{k^{\prime}}\right)\left\langle\partial_{x} Q_{k^{\prime}}, \partial_{x} Q_{k}\right\rangle \\
& -\sum_{k^{\prime} \neq k}\left(\ell_{k^{\prime}} \dot{z}_{k^{\prime}}\right)\left\langle\partial_{x}^{2} Q_{k^{\prime}}, \partial_{x} Q_{k}\right\rangle .
\end{aligned}
$$

Thus, using the equation of $Q,(3.4),(3.8)$ and (3.18), we obtain

$$
\begin{aligned}
\left\langle\operatorname{Mod}_{\eta}, \partial_{x} Q_{k}\right\rangle= & c_{1}\left(\dot{\ell}_{k}+2 \alpha \ell_{k}\right)+O\left(\sum_{k^{\prime} \neq k}\left|\dot{\ell}_{k^{\prime}}+2 \alpha \ell_{k^{\prime}}\right| e^{-\frac{3}{4}\left|z_{k^{\prime}}-z_{k}\right|}\right) \\
& +O\left(\|\vec{\varepsilon}\|_{H^{1} \times L^{2}}^{2}+\sum_{l=1}^{K}\left|\ell_{l}\right|^{2}\right) .
\end{aligned}
$$

Note that, by (3.18) and the Cauchy-Schwarz inequality, we obtain

$$
\left|\left\langle\eta, \dot{z}_{k} \partial_{x}^{2} Q_{k}\right\rangle\right| \lesssim\left(\left|\dot{z}_{k}-\ell_{k}\right|+\left|\ell_{k}\right|\right)\|\vec{\varepsilon}\|_{H^{1} \times L^{2}} \lesssim\|\vec{\varepsilon}\|_{H^{1} \times L^{2}}^{2}+\sum_{k=1}^{K}\left|\ell_{k}\right|^{2} .
$$

Gathering above estimates and using (3.9)-(3.10)-(3.11), we obtain

$$
\begin{aligned}
& \left|\dot{\ell}_{k}+2 \alpha \ell_{k}+\kappa \sigma_{k}\left[\sigma_{k-1} e^{-\left(z_{k}-z_{k-1}\right)}-\sigma_{k+1} e^{-\left(z_{k+1}-z_{k}\right)}\right]\right| \\
& \lesssim\|\vec{\varepsilon}\|_{H^{1} \times L^{2}}^{2}+\sum_{l=1}^{K}\left|\ell_{l}\right|^{2}+\sum_{k^{\prime} \neq k}\left|\dot{\ell}_{k^{\prime}}+2 \alpha \ell_{k^{\prime}}\right| e^{-\frac{3}{4}\left|z_{k^{\prime}}-z_{k}\right|}+\sum_{l=1}^{K-1} e^{-\theta\left(z_{l+1}-z_{l}\right)} .
\end{aligned}
$$

We obtain (3.23) by combining (3.24) for all $k \in\{1, \ldots, K\}$.

3.3. Energy estimates. For $\mu>0$ small to be chosen, we denote $\rho=2 \alpha-\mu$. Consider the nonlinear energy functional

$$
\mathcal{E}=\int\left\{\left(\partial_{x} \varepsilon\right)^{2}+(1-\rho \mu) \varepsilon^{2}+(\eta+\mu \varepsilon)^{2}-2[F(R+\varepsilon)-F(R)-f(R) \varepsilon]\right\} .
$$

We recall the following energy estimates.

Lemma 3.5. There exists $\mu>0$ such that in the context of Lemma 3.2, the following hold.

(i) Coercivity and bound.

$$
\mu\|\vec{\varepsilon}\|_{H^{1} \times L^{2}}^{2}-\frac{1}{2 \mu} \sum_{k=1}^{K}\left(\left(a_{k}^{+}\right)^{2}+\left(a_{k}^{-}\right)^{2}\right) \leq \mathcal{E} \leq \frac{1}{\mu}\|\vec{\varepsilon}\|_{H^{1} \times L^{2}}^{2} .
$$

(ii) Time variation.

$$
\frac{\mathrm{d}}{\mathrm{d} t} \mathcal{E} \leq-2 \mu \mathcal{E}+\frac{1}{\mu}\|\vec{\varepsilon}\|_{H^{1} \times L^{2}}\left[\|\vec{\varepsilon}\|_{H^{1} \times L^{2}}^{2}+\sum_{k=1}^{K}\left|\ell_{k}\right|^{2}+\sum_{k=1}^{K-1} e^{-\left(z_{k+1}-z_{k}\right)}\right] .
$$

Remark 3.6. The above lemma is valid for any $\mu>0$ small enough. For future needs, we fix such a $\mu>0$ satisyfing the following additional smallness condition

$$
\mu \leq \min \left(1, \alpha,\left|\nu_{-}\right|\right) \text {. }
$$

Remark 3.7. In the case where $K=1$, the term $\sum_{k=1}^{K-1}$ due to the nonlinear interactions does not appear in (3.26). 
Proof. See [6, proof of Lemma 2.4].

3.4. Time evolution analysis. We introduce new parameters and functionals to analyse the time evolution of solutions in the framework of Lemma 3.2 First, we set, for $k=1, \ldots, K$,

and for $k=1, \ldots, K-1$ (when $K \geq 2$ ),

$$
y_{k}=z_{k}+\frac{\ell_{k}}{2 \alpha}
$$

$$
r_{k}=y_{k+1}-y_{k} \gg 1 \text {. }
$$

Second, we define

$$
\begin{aligned}
& \mathcal{K}_{+}=\left\{k=1, \ldots, K-1: \sigma_{k}=\sigma_{k+1}\right\}, \quad F_{+}=\sum_{k \in \mathcal{K}_{+}} e^{-r_{k}}, \\
& \mathcal{K}_{-}=\left\{k=1, \ldots, K-1: \sigma_{k}=-\sigma_{k+1}\right\}, \quad F_{-}=\sum_{k \in \mathcal{K}_{-}} e^{-r_{k}} .
\end{aligned}
$$

Third, we introduce notation for the damped components of the solution

$$
\mathcal{F}=\mathcal{E}+\mathcal{G}, \quad \mathcal{G}=\sum_{k=1}^{K}\left|\ell_{k}\right|^{2}+\frac{1}{2 \mu} \sum_{k=1}^{K}\left(a_{k}^{-}\right)^{2},
$$

and for all the components except distances

$$
\mathcal{N}=\left[\|\vec{\varepsilon}\|_{H^{1} \times L^{2}}^{2}+\sum_{k=1}^{K}\left|\ell_{k}\right|^{2}\right]^{\frac{1}{2}} .
$$

Last, we define

$$
b=\sum_{k=1}^{K}\left(a_{k}^{+}\right)^{2}, \quad \mathcal{M}=\frac{1}{\mu^{2}}\left(\mathcal{F}-\frac{b}{2 \nu^{+}}\right) .
$$

We rewrite the estimates of Lemmas 3.2 and 3.5 using such notation.

Lemma 3.8. Assume $K \geq 2$. Let any $1<\theta<\min \left(p-1, \frac{5}{4}\right)$. In the context of Lemma 3.2, the following hold.

(i) Comparison with original variables. For $k=1, \ldots, K-1$,

$$
\begin{gathered}
\left|r_{k}-\left(z_{k+1}-z_{k}\right)\right| \lesssim \mathcal{N}, \\
\mu \mathcal{N}^{2} \leq \mu\|\vec{\varepsilon}\|_{H^{1} \times L^{2}}^{2}+\sum_{l=1}^{K}\left|\ell_{l}\right|^{2} \leq \mathcal{F}+\frac{b}{2 \mu} \lesssim \mathcal{N}^{2} .
\end{gathered}
$$

(ii) ODE for the distances between solitary waves. The equation for the evolution of $y_{k}$ is

$$
\begin{aligned}
& \begin{array}{l}
\left\{\begin{array}{l}
\dot{y}_{1}=\frac{\kappa}{2 \alpha} \sigma_{1} \sigma_{2} e^{-r_{1}}+O\left(\mathcal{N}^{2}+F_{+}^{\theta}+F_{-}^{\theta}\right), \\
\dot{y}_{k}=-\frac{\kappa}{2 \alpha} \sigma_{k-1} \sigma_{k} e^{-r_{k-1}}+\frac{\kappa}{2 \alpha} \sigma_{k} \sigma_{k+1} e^{-r_{k}}+O\left(\mathcal{N}^{2}+F_{+}^{\theta}\right. \\
\dot{y}_{K}=-\frac{\kappa}{2 \alpha} \sigma_{K-1} \sigma_{K} e^{-r_{K-1}}+O\left(\mathcal{N}^{2}+F_{+}^{\theta}+F_{-}^{\theta}\right),
\end{array}\right. \\
\text { for } 2 \leq k \leq K-1 . \text { Moreover, there exists } \lambda>0 \text { such } \\
\frac{\mathrm{d}}{\mathrm{d} t}\left[\frac{1}{F_{+}}\right] \leq-\lambda+\frac{1}{\lambda F_{+}}\left(\mathcal{N}^{2}+F_{+}^{\theta}+F_{-}^{\theta}\right),
\end{array} \\
& \frac{\mathrm{d}}{\mathrm{d} t}\left[\frac{1}{F_{-}}\right] \geq \lambda-\frac{1}{\lambda F_{-}}\left(\mathcal{N}^{2}+F_{+}^{\theta}+F_{-}^{\theta}\right) .
\end{aligned}
$$

(iii) Exponential instability.

$$
\left|\dot{b}-2 \nu^{+} b\right| \lesssim \mathcal{N}^{3}+\mathcal{N}\left(F_{+}+F_{-}\right) .
$$


(iv) Damped components.

$$
\begin{aligned}
& \frac{\mathrm{d}}{\mathrm{d} t} \mathcal{F}+2 \mu \mathcal{F} \lesssim \mathcal{N}^{3}+\mathcal{N}\left(F_{+}+F_{-}\right), \\
& \frac{\mathrm{d}}{\mathrm{d} t} \mathcal{G}+2 \mu \mathcal{G} \lesssim \mathcal{N}^{3}+\mathcal{N}\left(F_{+}+F_{-}\right) .
\end{aligned}
$$

(v) Liapunov type functional.

$$
\frac{\mathrm{d}}{\mathrm{d} t} \mathcal{M} \leq-\mathcal{N}^{2}+C\left(F_{+}^{2}+F_{-}^{2}\right) .
$$

(vi) Refined estimates for the distance. Setting

$$
R_{+}=\frac{1}{F_{+}} \exp \left(\lambda^{-1} \mathcal{M}\right) \quad \text { and } \quad R_{-}=\frac{1}{F_{-}} \exp \left(-3 \lambda^{-1} \mathcal{M}\right),
$$

where $\lambda$ is given in (ii), it holds

$$
\begin{aligned}
& \frac{\mathrm{d}}{\mathrm{d} t} R_{+} \leq\left(-\lambda+\frac{2}{\lambda F_{+}}\left(F_{+}^{\theta}+F_{-}^{\theta}\right)\right) \exp \left(\lambda^{-1} \mathcal{M}\right) \\
& \frac{\mathrm{d}}{\mathrm{d} t} R_{-} \geq\left(\lambda+\frac{2}{\lambda F_{-}} \mathcal{N}^{2}-\frac{2}{\lambda F_{-}}\left(F_{+}^{\theta}+F_{-}^{\theta}\right)\right) \exp \left(-3 \lambda^{-1} \mathcal{M}\right) .
\end{aligned}
$$

Remark 3.9. When using the quantities $F_{ \pm}$and $R_{ \pm}$, we tacitly assume that $\mathcal{K}_{ \pm}$ is not empty. Otherwise, the respective quantities are ignored. For example, if the set $\mathcal{K}_{+}$is empty, then (3.37) rewrites:

$$
\frac{\mathrm{d}}{\mathrm{d} t} R_{-} \geq\left(\lambda+\frac{2}{\lambda} \frac{\mathcal{N}^{2}}{F_{-}}-\frac{2}{\lambda} F_{-}^{\theta-1}\right) \exp \left(-3 \lambda^{-1} \mathcal{M}\right)
$$

When $K=1$, the quantities $F_{ \pm}$and $R_{ \pm}$are systematically 0 and the variables $r_{k}$ are not defined. The estimates (3.29), (3.33), (3.34) and (3.35) hold in this context.

Proof. Proof of (i): (3.28) follows from triangle inequality and the definition of $\mathcal{N}$ and (3.29) follows directly from (3.25).

Proof of (ii). In this computation, we use the convention that terms involving $y_{k}$ or $\sigma_{k}$ for $k \leq 0$ or $k \geq K+1$ are zero, for example by setting $\sigma_{0}=\sigma_{K+1}=0$. Similarly, when $e^{-r_{0}}$ and $e^{-r_{K}}$ appears, the corresponding term has to be ignored. By direct computation and using (3.18), (3.22)-(3.23), we obtain for $k=1, \ldots, K$,

$$
\dot{y}_{k}=\dot{z}_{k}+\frac{\dot{\ell}_{k}}{2 \alpha}=-\frac{\kappa}{2 \alpha} \sigma_{k-1} \sigma_{k} e^{-r_{k-1}}+\frac{\kappa}{2 \alpha} \sigma_{k} \sigma_{k+1} e^{-r_{k}}+O\left(\mathcal{N}^{2}+F_{+}^{\theta}+F_{-}^{\theta}\right) .
$$

It follows that, for any $k=1, \ldots, K-1$,

$$
\begin{aligned}
\dot{r}_{k}=\dot{y}_{k+1}-\dot{y}_{k}= & -\frac{\kappa}{\alpha} \sigma_{k} \sigma_{k+1} e^{-r_{k}}+\frac{\kappa}{2 \alpha} \sigma_{k+1} \sigma_{k+2} e^{-r_{k+1}}+\frac{\kappa}{2 \alpha} \sigma_{k-1} \sigma_{k} e^{-r_{k-1}} \\
& +O\left(\mathcal{N}^{2}+F_{+}^{\theta}+F_{-}^{\theta}\right) .
\end{aligned}
$$

On the right-hand side of the above expression, the first term is always present for $k=1, \ldots, K-1$, while the second and third terms might be zero depending on the value of $k$. For $k \in \mathcal{K}_{+}$, it holds $\sigma_{k}=\sigma_{k+1}$ and one sees that

$$
\dot{r}_{k}=-\frac{\kappa}{\alpha} e^{-r_{k}}+\frac{\kappa}{2 \alpha} \sigma_{k+1} \sigma_{k+2} e^{-r_{k+1}}+\frac{\kappa}{2 \alpha} \sigma_{k-1} \sigma_{k} e^{-r_{k-1}}+O\left(\mathcal{N}^{2}+F_{+}^{\theta}+F_{-}^{\theta}\right),
$$

with the same observation concerning the second and third terms on the right-hand side. Thus,

$$
\sum_{k \in \mathcal{K}_{+}} \dot{r}_{k} e^{-r_{k}}=-\frac{\kappa}{2 \alpha} S_{+}+O\left(F_{+}\left(\mathcal{N}^{2}+F_{+}^{\theta}+F_{-}^{\theta}\right)\right)
$$

where $S_{+}$denotes

$$
S_{+}=\sum_{k \in \mathcal{K}_{+}}\left[2 e^{-2 r_{k}}-\sigma_{k+1} \sigma_{k+2} e^{-\left(r_{k}+r_{k+1}\right)}-\sigma_{k-1} \sigma_{k} e^{-\left(r_{k-1}+r_{k}\right)}\right] .
$$


We claim that there exists $\tilde{\lambda}>0$ such that $S_{+}$satisfies

$$
S_{+} \geq \tilde{\lambda} \sum_{k \in \mathcal{K}_{+}} e^{-2 r_{k}}
$$

Indeed, first, recall that the symmetric matrix of size $n$

$$
A_{n}=\left(\begin{array}{ccccc}
2 & -1 & 0 & \ldots & 0 \\
-1 & 2 & -1 & \ddots & \vdots \\
0 & -1 & 2 & \ddots & 0 \\
\vdots & \ddots & \ddots & \ddots & -1 \\
0 & \ldots & 0 & -1 & 2
\end{array}\right)
$$

is definite positive by the Sylvester criterion since for any $j \in\{1, \ldots n\}$, the $j$ th leading principal minor of this matrix, i.e. the determinant of its upper-left $j \times j$ sub-matrix, is positive (its value is $j+1$ ).

Second, observe that in the sum defining $S_{+}$, for given $k \in \mathcal{K}_{+}$, if $k-1 \notin \mathcal{K}_{+}$, then $\sigma_{k-1} \sigma_{k}=1$ (if $k \geq 2$ ) or 0 (if $k=1$ ) and thus the corresponding term is positive or zero and can be ignored in establishing a lower bound for $S_{+}$. The same property is true for the term corresponding to $\sigma_{k+1} \sigma_{k+2}$ if $k+1 \notin \mathcal{K}_{+}$. Letting $n=\operatorname{card}\left(\mathcal{K}_{+}\right)$, this observation justifies that $S_{+}$is lower bounded by the quadratic form associated to the matrix $A_{n}$ taken at the vector of $\mathbb{R}^{n}$ of components $\left\{e^{-r_{k}}: k \in \mathcal{K}_{+}\right\}$. This shows that (3.39) holds for some $\tilde{\lambda}>0$.

It follows from (3.38) and (3.39) that there exists $\lambda>0$ such that

$$
\sum_{k \in \mathcal{K}_{+}} \dot{r}_{k} e^{-r_{k}} \leq-\lambda F_{+}^{2}+\frac{1}{\lambda} F_{+}\left(\mathcal{N}^{2}+F_{+}^{\theta}+F_{-}^{\theta}\right)
$$

Thus,

$$
\frac{\mathrm{d}}{\mathrm{d} t}\left[\frac{1}{F_{+}}\right]=\frac{1}{F_{+}^{2}} \sum_{k \in \mathcal{K}_{+}} \dot{r}_{k} e^{-r_{k}} \leq-\lambda+\frac{1}{\lambda F_{+}}\left(\mathcal{N}^{2}+F_{+}^{\theta}+F_{-}^{\theta}\right) .
$$

The estimate (3.32) concerning $\frac{\mathrm{d}}{\mathrm{d} t}\left[\frac{1}{F_{-}}\right]$is proved similarly.

Proof of (iii). It follows from (3.21) and the bound $\left|a_{k}^{+}\right| \lesssim\|\vec{\varepsilon}\|_{H^{1} \times L^{2}} \leq \mathcal{N}$.

Proof of (iv). By the definition of $\mathcal{F}$ and (3.19), (3.21), (3.26), we have

$$
\begin{aligned}
\frac{\mathrm{d}}{\mathrm{d} t} \mathcal{F} & =\frac{\mathrm{d}}{\mathrm{d} t} \mathcal{E}+2 \sum_{k=1}^{K} \dot{\ell}_{k} \ell_{k}+\frac{1}{\mu} \sum_{k=1}^{K} \dot{a}_{k}^{-} a_{k}^{-} \\
& \leq-2 \mu \mathcal{E}-4 \alpha \sum_{k=1}^{K} \ell_{k}^{2}+\frac{\nu^{-}}{\mu} \sum_{k=1}^{K}\left(a_{k}^{-}\right)^{2}+O\left(\mathcal{N}^{3}+\mathcal{N}\left(F_{+}+F_{-}\right)\right)
\end{aligned}
$$

Since $0<\mu \leq \alpha$ and $0<\mu \leq\left|\nu^{-}\right|$(see (3.27), we obtain (3.34) for $\mathcal{F}$. The proof for $\mathcal{G}$ is the same.

Proof of (v). From (3.33) and (3.34), we estimate

$$
\frac{\mathrm{d}}{\mathrm{d} t} \mathcal{M}=\frac{1}{\mu^{2}}\left(\frac{\mathrm{d}}{\mathrm{d} t} \mathcal{F}-\frac{1}{2 \nu^{+}} \frac{\mathrm{d}}{\mathrm{d} t} b\right) \leq-\frac{2}{\mu}\left(\mathcal{F}+\frac{b}{2 \mu}\right)+O\left(\mathcal{N}^{3}+\mathcal{N}\left(F_{+}+F_{-}\right)\right) .
$$

Thus, from (3.29) and then for $\mathcal{N}$ small enough,

$$
\frac{\mathrm{d}}{\mathrm{d} t} \mathcal{M} \leq-2 \mathcal{N}^{2}+O\left(\mathcal{N}^{3}+\mathcal{N}\left(F_{+}+F_{-}\right)\right) \leq-\mathcal{N}^{2}+O\left(F_{+}^{2}+F_{-}^{2}\right) .
$$


Proof of (vi). From (3.31) and (3.35), we estimate

$$
\begin{aligned}
\frac{\mathrm{d}}{\mathrm{d} t} R_{+} & =\left(\frac{\mathrm{d}}{\mathrm{d} t}\left[\frac{1}{F_{+}}\right]+\frac{1}{\lambda F_{+}} \frac{\mathrm{d}}{\mathrm{d} t} \mathcal{M}\right) \exp \left(\lambda^{-1} \mathcal{M}\right) \\
& \leq\left(-\lambda+\frac{1}{\lambda F_{+}}\left(F_{+}^{\theta}+F_{-}^{\theta}+C F_{+}^{2}+C F_{-}^{2}\right)\right) \exp \left(\lambda^{-1} \mathcal{M}\right)
\end{aligned}
$$

which implies (3.36) . The estimate for $\frac{\mathrm{d}}{\mathrm{d} t} R_{-}$is proved similarly. Note that the coefficient 3 of the factor $\exp \left(-3 \lambda^{-1} \mathcal{M}\right)$ in the definition $R_{-}$allows us to obtain a positive factor $\mathcal{N}^{2}$ in the right-hand side of (3.37).

\subsection{Long-time energy asymptotics.}

Lemma 3.10. Let any $1<\theta<\min \left(p-1, \frac{5}{4}\right)$. In the context of Lemmas 3.2 and 3.8, it holds

$$
E(\vec{u})=K E(Q, 0)-c_{1} \kappa F_{+}+c_{1} \kappa F_{-}+O\left(\mathcal{N}^{2}+F_{+}^{\theta}+F_{-}^{\theta}\right) .
$$

Proof. Expanding $E\left(u, \partial_{t} u\right)$ using the decomposition (3.15), integration by parts, the equation $-\partial_{x}^{2} Q+Q-f(Q)=0$ and the definition of $G$ in (3.3), we find

$$
\begin{aligned}
2 E\left(u, \partial_{t} u\right)= & \int\left|\partial_{t} u\right|^{2}+2 E(R, 0)-2 \int G \varepsilon \\
& +\int\left(\left|\partial_{x} \varepsilon\right|^{2}+\varepsilon^{2}-2 F(R+\varepsilon)+2 F(R)+2 f(R) \varepsilon\right) .
\end{aligned}
$$

Thus, using (3.6), the Cauchy-Schwarz and Sobolev inequalities, it holds

$$
2 E\left(u, \partial_{t} u\right)=\int\left|\partial_{t} u\right|^{2}+2 E(R, 0)+O\left(\mathcal{N}^{2}+F_{+}^{2}+F_{-}^{2}\right) .
$$

Note also that (3.15) implies

$$
\int\left|\partial_{t} u\right|^{2} \lesssim \int\left(|\eta|^{2}+\sum_{k=1}^{K}\left|\ell_{k} \partial_{x} Q_{k}\right|^{2}\right) \lesssim \mathcal{N}^{2}
$$

Then, by direct computation, next $-\partial_{x}^{2} Q+Q-f(Q)=0$ and (3.5),

$$
\begin{aligned}
E(R, 0)= & K E(Q, 0)+\sum_{k<k^{\prime}} \int\left[\left(\partial_{x} Q_{k}\right)\left(\partial_{x} Q_{k^{\prime}}\right)+Q_{k} Q_{k^{\prime}}-f\left(Q_{k}\right) Q_{k^{\prime}}-f\left(Q_{k^{\prime}}\right) Q_{k}\right] \\
& -\int\left(F(R)-\sum_{k=1}^{K} F\left(Q_{k}\right)-\sum_{k \neq k^{\prime}} f\left(Q_{k}\right) Q_{k^{\prime}}\right) \\
= & K E(Q, 0)-\sum_{k<k^{\prime}}\left\langle f\left(Q_{k}\right), Q_{k^{\prime}}\right\rangle+O\left(F_{+}^{\theta}+F_{-}^{\theta}\right) .
\end{aligned}
$$

Last, from (3.7), (3.28) and the definition of $F_{+}$and $F_{-}$, we observe that

$$
\sum_{k<k^{\prime}}\left\langle f\left(Q_{k}\right), Q_{k^{\prime}}\right\rangle=c_{1} \kappa F_{+}-c_{1} \kappa F_{-}+O\left(\mathcal{N}^{2}+F_{+}^{\theta}+F_{-}^{\theta}\right)
$$

Indeed, in the above double sum $\sum_{k<k^{\prime}}$ in $k$ and $k^{\prime}$, the terms corresponding to $k^{\prime}=k+1$ contribute to $\pm c_{1} \kappa F_{ \pm}$(depending on $k \in \mathcal{K}_{ \pm}$) and the other terms (i.e. $k^{\prime} \geq k+2$ ) only contribute to the error term (see also (3.13)).

Gathering all the above estimates, we have proved (3.40).

Combining (3.40) with (1.3) and (2.14), we obtain the following result.

Corollary 3.11. Let $\vec{u}$ be a global solution of (1.1) satisfying the decomposition given by Lemma 3.2 on $[t, \infty)$, for some $t \geq 0$. Let $1<\theta<\min \left(p-1, \frac{5}{4}\right)$. Then

$$
2 \alpha \int_{t}^{\infty}\left\|\partial_{t} u(s)\right\|_{L^{2}}^{2} \mathrm{~d} s=-c_{1} \kappa F_{+}(t)+c_{1} \kappa F_{-}(t)+O\left(\mathcal{N}^{2}(t)+F_{+}^{\theta}(t)+F_{-}^{\theta}(t)\right) .
$$


3.6. General estimates for global solutions. The following result is similar to Proposition 3.1 in [6]. We repeat the proof for the sake of completeness.

Proposition 3.12. There exists a universal constant $\delta_{1}>0$ such that the following holds. Let $0<\delta<\delta_{1}$ and $\vec{u}$ be a global solution of (1.1) satisfying (2.13) with $K \geq 1$. Let $T_{\delta} \gg 1$ be such that $\vec{u}$ admits a decomposition as in Lemma 3.2 in a neighborhood of $T_{\delta}$, with

$$
\left[\mathcal{N}\left(T_{\delta}\right)\right]^{2}+b\left(T_{\delta}\right)+F_{+}\left(T_{\delta}\right)+F_{-}\left(T_{\delta}\right) \leq \delta^{2} .
$$

Then, for all $t \geq T_{\delta}$, it holds

$$
[\mathcal{N}(t)]^{2}+b(t)+F_{+}(t)+F_{-}(t) \lesssim \delta^{2}
$$

and

$$
F_{-}(t) \leq 3\left(\delta^{-2}+\lambda\left(t-T_{\delta}\right)\right)^{-1} .
$$

Proof. For a constant $C>1$ to be taken large enough, we introduce the following bootstrap estimates

$$
\mathcal{N} \leq C \delta, \quad b \leq C \delta^{2}, \quad F_{+} \leq \delta^{\frac{3}{2}}, \quad F_{-} \leq \delta^{\frac{3}{2}},
$$

and we set

$$
T_{*}=\sup \left\{t \in\left[T_{\delta}, \infty\right) \text { such that }(3.45) \text { holds on }\left[T_{\delta}, t\right]\right\}>T_{\delta} .
$$

We prove $T_{*}=\infty$ by strictly improving the bootstrap estimate (3.45) on $\left[T_{\delta}, T_{*}\right.$ ) for $C>1$ large enough. In the rest of the proof, the implicit constants in $\lesssim$ or $O(\cdot)$ do not depend on the constant $C$ of the bootstrap estimate (3.45).

Estimate on $\mathcal{N}$. From (3.34) and (3.45), it holds on $\left[T_{\delta}, T_{*}\right)$,

$$
\frac{\mathrm{d}}{\mathrm{d} t}\left[e^{2 \mu t} \mathcal{F}\right] \lesssim C^{3} \delta^{3} e^{2 \mu t}+C \delta^{\frac{5}{2}} e^{2 \mu t} \leq \delta^{2} e^{2 \mu t},
$$

for $\delta>0$ small enough (depending on $C$ ). From (3.29) and (3.42), $\mathcal{F}\left(T_{\delta}\right) \lesssim \delta^{2}$. Thus, integrating the above estimate on $\left[T_{\delta}, t\right]$, for any $t \in\left[T_{\delta}, T_{*}\right), \mathcal{F}(t) \lesssim \delta^{2}$. In particular, by (3.29), we obtain

$$
\|\vec{\varepsilon}\|_{H^{1} \times L^{2}}^{2} \lesssim \mathcal{F}+b \lesssim C \delta^{2} .
$$

Arguing similarly for the quantity $\mathcal{G}$, we have

$$
\sum_{k=1}^{K}\left|\ell_{k}\right|^{2}+\sum_{k=1}^{K}\left(a_{k}^{-}\right)^{2} \lesssim \mathcal{G} \lesssim \delta^{2} .
$$

Hence we obtain, for all $t \in\left[T_{\delta}, T_{*}\right)$,

$$
\mathcal{N}(t) \lesssim \sqrt{C} \delta
$$

For $C$ large enough, this strictly improves the bootstrap estimate (3.45) on $\mathcal{N}$ on the interval $\left[T_{\delta}, T_{*}\right)$.

Estimate on $b$. Now, we prove that for $C$ large enough, it holds for all $t \in\left[T_{\delta}, T_{*}\right)$,

$$
b(t) \leq \frac{C}{2} \delta^{2} .
$$

From (3.41) in Corollary 3.11 and (3.42), we have

$$
\int_{T_{\delta}}^{\infty}\left\|\partial_{t} u(s)\right\|_{L^{2}}^{2} \mathrm{~d} s \lesssim \mathcal{N}^{2}\left(T_{\delta}\right)+F_{+}\left(T_{\delta}\right)+F_{-}\left(T_{\delta}\right) \lesssim \delta^{2} .
$$

By (3.42), we have $b\left(T_{\delta}\right) \leq \delta^{2}$. For the sake of contradiction, take $C>4$ large and assume that there exists $t_{2} \in\left[T_{\delta}, T_{*}\right)$ such that

$$
b\left(t_{2}\right)=\frac{C}{2} \delta^{2}, \quad b(t)<\frac{C}{2} \delta^{2} \quad \text { on }\left[T_{\delta}, t_{2}\right) .
$$


On the one hand, by continuity of $b$, there exists $t_{1} \in\left[T_{\delta}, t_{2}\right)$ such that

$$
b\left(t_{1}\right)=\frac{C}{4} \delta^{2} \quad \text { and } \quad b(t)>\frac{C}{4} \delta^{2} \quad \text { on }\left(t_{1}, t_{2}\right] .
$$

Using (3.33) and the bootstrap estimates (3.45), we have

$$
\frac{\mathrm{d}}{\mathrm{d} t} b=2 \nu^{+} b+O\left(C^{3} \delta^{3}+C \delta^{\frac{5}{2}}\right)
$$

which implies (for $\delta$ small enough depending on $C$ )

$$
t_{2}-t_{1}=\frac{\log 2}{2 \nu^{+}}+O\left(\delta^{\frac{1}{3}}\right)
$$

and thus

$$
\int_{t_{1}}^{t_{2}} b(s) \mathrm{d} s \gtrsim C \delta^{2} .
$$

On the other hand, by (3.2), (3.15), (3.46), (3.49) and (3.50),

$$
\int_{t_{1}}^{t_{2}}\|\eta(t)\|_{L^{2}}^{2} \mathrm{~d} t \lesssim \int_{t_{1}}^{t_{2}}\left(\left\|\partial_{t} u(t)\right\|_{L^{2}}^{2}+\sum_{k=1}^{K}\left\|\ell_{k} \partial_{x} Q_{k}(t)\right\|_{L^{2}}^{2}\right) \mathrm{d} t \lesssim \delta^{2} .
$$

By the definition of $a_{k}^{ \pm}$, one has

$$
a_{k}^{+}=\zeta^{+}\left\langle\varepsilon, Y_{k}\right\rangle+\left\langle\eta, Y_{k}\right\rangle, \quad a_{k}^{-}=\zeta^{-}\left\langle\varepsilon, Y_{k}\right\rangle+\left\langle\eta, Y_{k}\right\rangle
$$

and thus

$$
a_{k}^{+}=\frac{\zeta^{+}}{\zeta^{-}} a_{k}^{-}+\frac{\zeta^{-}-\zeta^{+}}{\zeta^{-}}\left\langle\eta, Y_{k}\right\rangle .
$$

Combining (3.46), (3.50) and (3.52), we find the estimate $\int_{t_{1}}^{t_{2}} b(s) \mathrm{d} s \lesssim \delta^{2}$, which contradicts (3.51) for $C$ large enough. This proves (3.48).

Estimate on $F_{-}$. Let $1<\theta<\min \left(p-1, \frac{5}{4}\right)$. First, from 3.41 in Corollary 3.11 and (3.45), we have

$$
F_{+} \leq 2 F_{-}+O\left(\mathcal{N}^{2}\right) \quad \text { and } \quad F_{+}^{\theta} \leq 2 F_{-}^{\theta}+\mathcal{N}^{2} .
$$

Second, from (3.37), (3.45) and (3.53), we have

$$
\begin{aligned}
\frac{\mathrm{d}}{\mathrm{d} t} R_{-} & \geq\left(\lambda+\frac{2}{\lambda F_{-}}\left(\mathcal{N}^{2}-F_{+}^{\theta}-F_{-}^{\theta}\right)\right) \exp \left(-3 \lambda^{-1} \mathcal{M}\right) \\
& \geq\left(\lambda-\frac{6}{\lambda} F_{-}^{\theta-1}\right) \exp \left(-3 \lambda^{-1} \mathcal{M}\right) \geq \frac{\lambda}{2} .
\end{aligned}
$$

By integration on $\left[T_{\delta}, t\right]$ for any $t \in\left[T_{\delta}, T_{*}\right)$, it holds

$$
R_{-}(t) \geq R_{-}\left(T_{\delta}\right)+\frac{\lambda}{2}\left(t-T_{\delta}\right)
$$

By the definition of $R_{-}$and (3.42), we have $R_{-}\left(T_{\delta}\right) \geq \frac{1}{2} \delta^{-2}$. Thus, (3.54) implies that, for any $t \in\left[T_{\delta}, T_{*}\right)$

$$
F_{-}(t) \leq \frac{3}{2 R_{-}} \leq \frac{3}{2}\left(\frac{1}{2} \delta^{-2}+\frac{\lambda}{2}\left(t-T_{\delta}\right)\right)^{-1} \leq 3 \delta^{2} .
$$

This is strictly improves the bootstrap estimate (3.45) of $F_{-}$on the interval $\left[T_{\delta}, T_{*}\right.$ ) and proves (3.44).

Estimate on $F_{+}$. From (3.47), (3.53) and (3.44), we observe that

$$
F_{+} \leq 2 F_{-}+O\left(\mathcal{N}^{2}\right) \leq O\left(C \delta^{2}\right) .
$$

For $\delta$ small enough (depending on $C$ ), this strictly improves the estimate (3.45) of $F_{+}$on $\left[T_{\delta}, T_{*}\right)$.

The previous estimates prove that $T_{*}=\infty$ and that (3.43) holds on $\left[T_{\delta}, \infty\right)$. 


\section{Alternate signs property for neighbor solitary WaVes}

In this Section, we prove the following property.

Proposition 4.1. Let $\vec{u}$ be a global solution of (1.1) such that $K \geq 2$ in (2.13) of Theorem 2.3. Then,

$$
\sigma_{k}=-\sigma_{k+1} \quad \text { for all } k \in\{1, \ldots, K-1\} .
$$

Proof. We perform computations in the context of Proposition 3.12 Assuming that $\mathcal{K}_{+}$is not empty, we reach a contradiction. In this context, if $\mathcal{K}_{-}$is empty, we have $F_{-}=0$. Otherwise, we may use the estimate (3.44) on $F_{-}$proved in Proposition 3.12 Using (3.36), (3.43) and (3.44), we obtain that for any $t \in\left[T_{\delta}, \infty\right)$,

$$
\frac{\mathrm{d}}{\mathrm{d} t} R_{+} \leq-\frac{3}{4} \lambda+\frac{3}{\lambda} F_{+}^{\theta-1}+\frac{2}{\lambda} R_{+} F_{-}^{\theta} \leq-\frac{\lambda}{2}+\frac{18}{\lambda}\left(\delta^{-2}+\lambda\left(t-T_{\delta}\right)\right)^{-\theta} R_{+} .
$$

Define the auxiliary function

$$
\widetilde{R}^{+}(t)=R_{+}(t) \exp \left[\frac{18}{\lambda^{2}(\theta-1)}\left(\delta^{-2}+\lambda\left(t-T_{\delta}\right)\right)^{1-\theta}\right], \quad \text { for } t \in\left[T_{\delta}, \infty\right) .
$$

By direct computation and (4.2), we observe that

$$
\frac{\mathrm{d}}{\mathrm{d} t} \widetilde{R}^{+} \leq-\frac{\lambda}{2} \exp \left[\frac{18}{\lambda^{2}(\theta-1)}\left(\delta^{-2}+\lambda\left(t-T_{\delta}\right)\right)^{1-\theta}\right] \leq-\frac{\lambda}{2} .
$$

By integrating the above estimate on $\left[T_{\delta}, t\right]$, we obtain

$$
\widetilde{R}^{+}(t) \leq \widetilde{R}^{+}\left(T_{\delta}\right)-\frac{\lambda}{2}\left(t-T_{\delta}\right)
$$

which is contradictory with $\widetilde{R}^{+}(t) \geq 0$ for large $t$. This means that $\mathcal{K}_{+}=\emptyset$ and so $\mathcal{K}_{-}=\{1, \ldots, K-1\}$ : the signs of the solitary waves alternate.

\section{Description of LONG-Time ASYMPtotics}

We consider any global solution $\vec{u}$ of (1.1). We prove Theorem 1.1 by considering separately the no soliton case, and then the cases $K=1$ and $K \geq 2$ in Theorem 2.3.

5.1. No soliton case. If $\vec{u}(t)$ converges to 0 as $t \rightarrow \infty$, as a consequence of (7) of Theorem 2.3 in [1], $\vec{u}$ converges exponentially to 0 in $H^{1} \times L^{2}$. Alternatively, one can use the energy functional

$$
\int\left\{\left(\partial_{x} u\right)^{2}+(1-\rho \mu) u^{2}+\left(\partial_{t} u+\mu u\right)^{2}\right\}
$$

where $\mu>0$ is small and $\rho=2 \alpha-\mu$ as in 3.3 to prove the exponential convergence.

5.2. Single soliton case. Assume that $\vec{u}$ follows the solitary wave scenario in Theorem 2.3 with $K=1$. Let $\delta>0$ to be chosen small enough. Following Lemma 3.2 and Proposition 3.12 there exists $T_{\delta}>0$ such that estimates (3.43)(3.44) hold on $\left[T_{\delta}, \infty\right)$. When $K=1$, by convention

$$
F_{+}(t)=F_{-}(t)=0, \quad \text { for } t \geq 0
$$

and the estimates proved in the previous Sections simplify; see Remarks 3.3, 3.7 and 3.9. In particular, from (3.33) and (3.34), it holds

and from (3.29) and (3.45),

$$
\begin{gathered}
\left|\dot{b}-2 \nu^{+} b\right| \lesssim \mathcal{N}^{3}, \\
\frac{\mathrm{d}}{\mathrm{d} t} \mathcal{F}+2 \mu \mathcal{F} \lesssim \mathcal{N}^{3}, \quad \frac{\mathrm{d}}{\mathrm{d} t} \mathcal{G}+2 \mu \mathcal{G} \lesssim \mathcal{N}^{3},
\end{gathered}
$$

$$
\mathcal{N}^{3} \leq C \delta \mathcal{N}^{2} \lesssim C \delta\left(\mathcal{F}+\frac{b}{2 \mu}\right)
$$


Set $\tilde{b}=b-\delta^{\frac{1}{2}} \mathcal{F}$, observe that

$$
\tilde{b}=\left(1+\frac{\delta^{\frac{1}{2}}}{2 \mu}\right) b-\delta^{\frac{1}{2}}\left(\mathcal{F}+\frac{b}{2 \mu}\right) \leq\left(1+\frac{\delta^{\frac{1}{2}}}{2 \mu}\right) b .
$$

Therefore, using (5.1), (5.2) and (5.3),

$$
\begin{aligned}
\frac{\mathrm{d}}{\mathrm{d} t} \tilde{b} & \geq 2 \nu^{+} b+2 \mu \delta^{\frac{1}{2}} \mathcal{F}-C \delta^{\frac{3}{4}}\left(\mathcal{F}+\frac{b}{2 \mu}\right) \\
& \geq\left(2 \nu^{+}-\delta^{\frac{1}{2}}\right) b+\left(2 \mu \delta^{\frac{1}{2}}-C \delta^{\frac{3}{4}}\right)\left(\mathcal{F}+\frac{b}{2 \mu}\right) \geq \nu^{+} \tilde{b},
\end{aligned}
$$

where $\delta>0$ small enough such that

$$
\left(2 \nu^{+}-\delta^{\frac{1}{2}}\right)\left(1+\frac{\delta^{\frac{1}{2}}}{2 \mu}\right) \geq \nu^{+}, \quad 2 \mu \delta^{\frac{1}{2}}-C \delta^{\frac{3}{4}}>0 .
$$

Integrating on $[t, s] \in\left[T_{\delta}, \infty\right)$,

$$
\tilde{b}(t) \leq e^{-\nu^{+}(s-t)} \tilde{b}(s) .
$$

Let $s \rightarrow \infty$ in (5.4) and using (3.45), we obtain

$$
\tilde{b}(t) \leq 0, \quad b(t) \leq \delta^{\frac{1}{2}} \mathcal{F}(t) \quad \text { for } t \geq T_{\delta} .
$$

Thus, using (5.1), (5.2) and (5.3) again,

$$
\begin{aligned}
\frac{\mathrm{d}}{\mathrm{d} t}\left(\mathcal{F}+\frac{b}{2 \mu}\right) & \leq-2 \mu \mathcal{F}+\frac{\nu^{+}}{\mu} b+C \delta^{\frac{3}{4}}\left(\mathcal{F}+\frac{b}{2 \mu}\right) \\
& \leq-\left(2 \mu-C \delta^{\frac{3}{4}}\right)\left(\mathcal{F}+\frac{b}{2 \mu}\right)+\left(1+\frac{\nu^{+}}{\mu}\right) b \leq-\mu\left(\mathcal{F}+\frac{b}{2 \mu}\right),
\end{aligned}
$$

by possibly choosing $\delta>0$ small enough. Integrating on $\left[T_{\delta}, t\right]$, we obtain

$$
\left(\mathcal{F}+\frac{b}{2 \mu}\right)(t) \leq e^{-\mu t} e^{\mu T_{\delta}}\left(\mathcal{F}+\frac{b}{2 \mu}\right)\left(T_{\delta}\right) \lesssim e^{-\mu t} e^{\mu T_{\delta}} \delta^{2} .
$$

Therefore, using again (3.29),

$$
\mathcal{N}^{2}(t) \lesssim\left(\mathcal{F}+\frac{b}{2 \mu}\right)(t) \lesssim e^{-\mu t} .
$$

From (3.18) and (5.5), we have $\left|\dot{z}_{1}\right| \leq e^{-\frac{\mu}{2} t}$, which proves that $z_{1}(t)$ converges exponentially to its limit as $t \rightarrow \infty$. In view of the decomposition of $\vec{u}$ in (3.15), the proof in the case $K=1$ is complete.

5.3. Multi-soliton case. Assume that $\vec{u}$ follows the multi-solitary wave scenario in Theorem 2.3 with $K \geq 2$. Let $\delta>0$ to be chosen small enough. Following Lemma 3.2 and Proposition 3.12 there exists $T_{\delta}>0$ such that estimates (3.43)(3.44) hold on $\left[T_{\delta}, \infty\right)$. Recall that from Proposition 4.1, the set $\mathcal{K}_{+}$is empty. In particular, following Remark 3.9, we use the estimates of Lemma 3.8 ignoring the quantity $F_{+}$. We start by showing that the quantity $\mathcal{N}(t)$ decays as $t^{-1}$.

Proposition 5.1. There exists $T>0$ such that the decomposition of $\vec{u}$ satisfies, for all $t \geq T$

$$
F_{-}(t) \lesssim t^{-1}, \quad \mathcal{N}(t) \lesssim t^{-1}
$$

Proof. The proof is inspired by that of [6, Proposition 3.2]. Let $0<\delta<\delta_{1}$ in the context of Proposition 3.12. From (3.43) and (3.44), there exists $T>0$ large enough (fix any $T \geq 4 T_{\delta}$ ) such that, for all $t \geq T / 2$,

$$
\mathcal{N}(t) \leq C \delta, \quad F_{-}(t) \leq \frac{6}{\lambda} t^{-1}
$$


In particular, from (3.33) and (3.34)

$$
\left|\frac{\mathrm{d} b}{\mathrm{~d} t}-2 \nu^{+} b\right| \lesssim \mathcal{N}^{3}+t^{-1} \mathcal{N}, \quad \frac{\mathrm{d}}{\mathrm{d} t} \mathcal{F}+2 \mu \mathcal{F} \lesssim \mathcal{N}^{3}+t^{-1} \mathcal{N} .
$$

Our goal is to obtain the decay rate of $\mathcal{N}$. The above bounds are not quite enough because of the term $\mathcal{N}^{3}$ for which only smallness is known at this point. This is the reason why we will work on a modification $\tilde{b}$ of $b$. Recall (3.29):

$$
0 \leq \mathcal{N}^{2} \lesssim \mathcal{F}+\frac{b}{2 \mu}
$$

For $0<\omega \ll 1$ to be chosen later, observe that, for $\delta$ small,

$$
\mathcal{N}^{3}+t^{-1} \mathcal{N} \lesssim \omega^{2} \mathcal{N}^{2}+\omega^{-2} t^{-2} \lesssim \omega^{2}\left(\mathcal{F}+\frac{b}{2 \mu}\right)+\omega^{-2} t^{-2}
$$

(Here and below the implied constants do not depend on $\omega$ ). Set $\tilde{b}=b-\omega \mathcal{F}$ and observe that

$$
\tilde{b}=b-\omega \mathcal{F}=\left(1+\frac{\omega}{2 \mu}\right) b-\omega\left(\mathcal{F}+\frac{1}{2 \mu} b\right) \leq\left(1+\frac{\omega}{2 \mu}\right) b .
$$

Therefore, using (5.7) and (5.8),

$$
\begin{aligned}
\frac{\mathrm{d} \tilde{b}}{\mathrm{~d} t} & \geq 2 \nu^{+} b+2 \omega \mu \mathcal{F}-C \omega^{2}\left(\mathcal{F}+\frac{b}{2 \mu}\right)-C \omega^{-2} t^{-2} \\
& \geq\left(2 \nu^{+}-\omega\right) b+\left(2 \omega \mu-C \omega^{2}\right)\left(\mathcal{F}+\frac{b}{2 \mu}\right)-C \omega^{-2} t^{-2} \\
& \geq \nu^{+} \tilde{b}-C \omega^{-2} t^{-2},
\end{aligned}
$$

where $\omega>0$ is taken small enough such that

$$
\left(2 \nu^{+}-\omega\right)\left(1+\frac{\omega}{2 \mu}\right)^{-1} \geq \nu^{+}, \quad 2 \omega \mu-C \omega^{2}>0 .
$$

Integrating on $[t, s] \subset[T / 2, \infty)$, we obtain

$$
\tilde{b}(t)-e^{-\nu^{+}(s-t)} \tilde{b}(s) \leq C \omega^{-2} \int_{t}^{s} e^{-\nu^{+}(\tau-t)} \tau^{-2} \mathrm{~d} \tau \lesssim \omega^{-2} t^{-2} .
$$

Let $s \rightarrow \infty$ in (5.9) and using (3.45), we obtain for all $t \geq T / 2$,

$$
\tilde{b}(t) \lesssim \omega^{-2} t^{-2}, \quad b(t) \leq C \omega^{-2} t^{-2}+\omega \mathcal{F}(t) .
$$

Thus using (5.7) and (5.8) again, it holds

$$
\begin{aligned}
\frac{\mathrm{d}}{\mathrm{d} t}\left(\mathcal{F}+\frac{b}{2 \mu}\right) & \leq-2 \mu \mathcal{F}+\frac{\nu^{+}}{\mu} b+C \omega^{2}\left(\mathcal{F}+\frac{b}{2 \mu}\right)+C \omega^{-2} t^{-2} \\
& \leq-\left(2 \mu-C \omega^{2}\right)\left(\mathcal{F}+\frac{b}{2 \mu}\right)+\left(1+\frac{\nu^{+}}{\mu}\right) b+C \omega^{-2} t^{-2} \\
& \leq-\mu\left(\mathcal{F}+\frac{b}{2 \mu}\right)+C \omega^{-2} t^{-2}
\end{aligned}
$$

by possibly choosing $\omega>0$ small enough. Integrating on $[T / 2, t]$, we obtain

$$
\left(\mathcal{F}+\frac{b}{2 \mu}\right)(t)-e^{-\mu\left(t-\frac{T}{2}\right)}\left(\mathcal{F}+\frac{b}{2 \mu}\right)(T / 2) \lesssim \int_{\frac{T}{2}}^{t} e^{-\mu(t-s)} s^{-2} \mathrm{~d} s
$$

Therefore, using again (3.29),

$$
\mathcal{N}^{2}(t) \lesssim\left(\mathcal{F}+\frac{b}{2 \mu}\right)(t) \lesssim t^{-2}+\delta^{2} e^{\mu T / 2} e^{-\mu t} \leq t^{-2},
$$

which proves (5.6). 
We continue the proof of Theorem 1.1. In view of the alternate signs property (4.1) and the decay estimate (5.6), the system (3.30) rewrites as, for $k=2, \cdots, K-1$, and any $t \geq T$

$$
\left\{\begin{array}{l}
\dot{y}_{1}=-\frac{\kappa}{2 \alpha} e^{-\left(y_{2}-y_{1}\right)}+O\left(t^{-\theta}\right), \\
\dot{y}_{k}=\frac{\kappa}{2 \alpha}\left(e^{-\left(y_{k}-y_{k-1}\right)}-e^{-\left(y_{k+1}-y_{k}\right)}\right)+O\left(t^{-\theta}\right), \\
\dot{y}_{K}=\frac{\kappa}{2 \alpha} e^{-\left(y_{K}-y_{K-1}\right)}+O\left(t^{-\theta}\right) .
\end{array}\right.
$$

This system of ODEs is studied in 16 and 8, where it appears naturally in a different context (the description of characteristic blowup points of the semilinear wave equation), with slightly different perturbation terms. For the convenience of the reader, we provide a study of the dynamics.

We introduce an explicit solution to the unperturbed ODE system (1.9)

$$
\bar{y}_{k}(t):=\left(k-\frac{K+1}{2}\right) \log t+\tau_{k}, \quad \text { for } k=1, \ldots, K,
$$

where $\left(\tau_{k}\right)_{k=1, \ldots, K}$ are constants uniquely defined by (1.8) (see also [8, p. 1549]). From (5.10), we observe that $\sum_{k=1}^{K} \dot{y}_{k}=O\left(t^{-\theta}\right)$. Since $\theta>1$, there exist $\bar{y}_{\infty} \in \mathbb{R}$ such that

$$
\frac{1}{K} \sum_{k=1}^{K} y_{k}=\bar{y}_{\infty}+O\left(t^{-\theta+1}\right) \text {. }
$$

We introduce, for $k=1, \ldots, K$,

$$
\xi_{k}(t):=y_{k}-\bar{y}_{k}-\bar{y}_{\infty} .
$$

Observe that, for $k=1, \cdots, K-1$,

$$
y_{k+1}-y_{k}=\xi_{k+1}-\xi_{k}+\log t+\left(\tau_{k+1}-\tau_{k}\right),
$$

so that using (1.8),

$$
\frac{\kappa}{2 \alpha} e^{-\left(y_{k+1}-y_{k}\right)}=\frac{\kappa}{2 \alpha} e^{-\left(\xi_{k+1}-\xi_{k}\right)-\log t-\left(\tau_{k+1}-\tau_{k}\right)}=\gamma_{k} t^{-1} e^{-\left(\xi_{k+1}-\xi_{k}\right)} .
$$

Therefore, from (5.10) and $\gamma_{k}-\gamma_{k-1}=\frac{K+1}{2}-k$, it holds $(2 \leq k \leq K-1)$

$$
\left\{\begin{array}{l}
\dot{\xi}_{1}=-t^{-1} \gamma_{1}\left(e^{-\left(\xi_{2}-\xi_{1}\right)}-1\right)+O\left(t^{-\theta}\right), \\
\dot{\xi}_{k}=t^{-1}\left(\gamma_{k-1}\left(e^{-\left(\xi_{k}-\xi_{k-1}\right)}-1\right)-\gamma_{k}\left(e^{-\left(\xi_{k+1}-\xi_{k}\right)}-1\right)\right)+O\left(t^{-\theta}\right), \\
\dot{\xi}_{K}=t^{-1} \gamma_{K-1}\left(e^{-\left(\xi_{K}-\xi_{K-1}\right)}-1\right)+O\left(t^{-\theta}\right) .
\end{array}\right.
$$

To complete the proof of Theorem 1.1, it suffices to show that for any $k=1, \ldots, K$,

$$
\xi_{k}(t)=O\left(t^{-\theta+1}\right) .
$$

First, we prove a bound on $\xi_{k}$.

Lemma 5.2. There exists $M>0$ such that for all $k=1, \ldots, K$ and for all $t \geq T_{\delta}$, it holds $\left|\xi_{k}(t)\right| \leq M$.

Proof. Set

$$
\zeta_{0}=\zeta_{K}=0 \quad \text { and } \quad \zeta_{k}=\xi_{k+1}-\xi_{k} \quad \text { for } k=1, \cdots, K-1 .
$$

From (5.13), it holds for $k=1, \ldots, K-1$ (with $\left.\gamma_{0}=\gamma_{K}=0\right)$

$$
\dot{\zeta}_{k}=t^{-1}\left(-\gamma_{k+1}\left(e^{-\zeta_{k+1}}-1\right)+2 \gamma_{k}\left(e^{-\zeta_{k}}-1\right)-\gamma_{k-1}\left(e^{-\zeta_{k-1}}-1\right)\right)+O\left(t^{-\theta}\right) .
$$

Claim 5.3. There exists $M_{1}>0$ such that for all $k=1, \ldots, K-1$, and for all $t \geq T_{\delta}$, it holds $\left|\zeta_{k}(t)\right| \leq M_{1}$. 
Proof Claim 5.3. First, we prove a lower bound $\zeta_{k}(t) \geq-M_{1}$, for some $M_{1}>0$. Fix $\vartheta=\frac{1+\theta}{2}$ and for $D_{1}>0$ to be fixed later, denote for $k=1, \cdots, K-1$,

$$
\rho_{k}=\gamma_{k}\left(e^{-\zeta_{k}}-1\right)+D_{1} t^{-\vartheta+1}, \quad \rho_{+}=\max _{k} \rho_{k}
$$

Let us prove that $\rho_{+}$is non increasing for large enough times using a bootstrap argument. For $C_{1}>0$ and $T_{1} \geq T_{\delta}$ to be chosen later, let

$$
T_{*}=\sup \left\{t \in\left[T_{1}, \infty\right) \text { such that } \rho_{+} \leq C_{1} \text { on }\left[T_{1}, t\right]\right\} .
$$

Let $t \in\left[T_{1}, T_{*}\right)$, and consider an index $k$ such that $\rho_{k}(t)=\rho_{+}(t)$. Observe that

$$
\begin{gathered}
\gamma_{k} e^{-\zeta_{k}(t)} \leq \rho_{k}(t)+\gamma_{k} \leq C_{1}+K^{2} \\
2 \gamma_{k}\left(e^{-\zeta_{k}(t)}-1\right) \geq \gamma_{k+1}\left(e^{-\zeta_{k+1}(t)}-1\right)+\gamma_{k-1}\left(e^{-\zeta_{k-1}(t)}-1\right) .
\end{gathered}
$$

Gathering (5.15), (5.16) and (5.17), there exist $C_{0}>0$ such that at $t$,

$$
\begin{aligned}
\dot{\rho}_{k}= & -\gamma_{k} e^{-\zeta_{k}} \dot{\zeta}_{k}-D_{1}(\vartheta-1) t^{-\vartheta} \\
= & -\gamma_{k} e^{-\zeta_{k}} t^{-1}\left(-\gamma_{k+1}\left(e^{-\zeta_{k+1}}-1\right)+2 \gamma_{k}\left(e^{-\zeta_{k}}-1\right)-\gamma_{k-1}\left(e^{-\zeta_{k-1}}-1\right)\right) \\
& +O\left(\gamma_{k} e^{-\tau_{k}(t)} t^{-\theta}\right)-D_{1}(\vartheta-1) t^{-\vartheta} \\
\leq & C_{0} \gamma_{k} e^{-\zeta_{k}(t)} t^{-\theta}-D_{1}(\vartheta-1) t^{-\vartheta} \\
\leq & \left(\left(C_{1}+K^{2}\right) C_{0}-D_{1}(\vartheta-1) T_{1}^{\theta-\vartheta}\right) t^{-\theta}
\end{aligned}
$$

Fix $T_{1} \geq T_{\delta}$ and then $D_{1}, C_{1}$ such that

$$
\begin{gathered}
(\vartheta-1) T_{1}^{\theta-\vartheta} \geq C_{0}+1, \\
D_{1} \geq C_{0}\left(\rho_{+}\left(T_{1}\right)+1+K^{2}\right)+1, \quad C_{1}=\rho_{+}\left(T_{1}\right)+D_{1}+1 .
\end{gathered}
$$

Then, there holds

$$
\begin{aligned}
\left(C_{1}+K^{2}\right) C_{0}-D_{1}(\vartheta-1) T_{1}^{\theta-\vartheta} & \leq\left(\rho_{+}\left(T_{1}\right)+D_{1}+1+K^{2}\right) C_{0}-D_{1}\left(C_{0}+1\right) \\
& \leq\left(\rho_{+}\left(T_{1}\right)+1+K^{2}\right) C_{0}-D_{1} \leq-1 .
\end{aligned}
$$

Since $\rho_{+}\left(T_{1}\right)<C_{1}$, by continuity, $T_{*}>T_{1}$. We also have $\dot{\rho}_{k}(t)<0$, so that $\rho_{k}$ is decreasing at $t$; note that this property holds for any index $k$ such that $\rho_{k}(t)=$ $\rho_{+}(t)$. If $j$ is an index such that $\rho_{j}(t)<\rho_{+}(t)$, then by continuity this inequality holds on a neighborhood of $t$. Thus $\rho_{+}$is decreasing at $t$, for any $t \in\left[T_{1}, T^{*}\right)$. In particular, $\rho_{+}(t) \leq \rho_{+}\left(T_{1}\right) \leq C_{1}$. By continuity, we obtain $T_{*}=\infty$ and so for all $t \geq T_{1}, \rho_{+}(t) \leq C_{1}$.

By continuity, there exists $C_{2}$ such that for all $t \geq T_{\delta}, \rho_{+}(t) \leq C_{2}$, and so for all $k=1, \ldots, K-1$, for all $t \geq T_{\delta}, e^{-\zeta_{k}(t)} \leq 2 C_{2}+1$, since $D_{1}>0$ and $\gamma_{k} \geq 1 / 2$. Therefore, for $M_{1}=\log \left(2 C_{2}+1\right)$, we have proved the lower bound on $\zeta_{k}(t)$.

Arguing similarly using the minimum of $\tilde{\rho}_{k}=\gamma_{k}\left(e^{-\zeta_{k}}-1\right)-D_{1} t^{-\vartheta+1}$, one also proves an upper bound on $\zeta_{k}$.

By (15.12) and $\sum_{k=1}^{K} \bar{y}_{k}=\sum_{k=1}^{K} \tau_{k}=0$, there exists $M_{2}>0$ such that for all $t \geq T_{\delta}$, $\left|\sum_{k=1}^{K} \xi_{k}(t)\right| \leq M_{2}$. By contradiction, assume that for some $k_{0}$ and $t \geq T_{\delta}$

$$
\xi_{k_{0}}(t) \geq M \quad \text { where } \quad M:=\frac{2 M_{2}}{K}+\frac{K-1}{2} M_{1}
$$

Then,

$$
\begin{aligned}
& \xi_{k}(t)=\xi_{k_{0}}(t)+\sum_{j=k_{0}}^{k-1} \zeta_{j} \geq M-\left|k-k_{0}\right| M_{1} \quad \text { for } 1 \leq k_{0}<k \leq K \\
& \xi_{k}(t)=\xi_{k_{0}}(t)-\sum_{j=k}^{k_{0}-1} \zeta_{j} \geq M-\left|k-k_{0}\right| M_{1} \quad \text { for } 1 \leq k<k_{0} \leq K
\end{aligned}
$$


so that (in view of the definition of $M$ )

$$
\sum_{k=1}^{K} \xi_{k}(t) \geq K M-M_{1} \sum_{k=1}^{K}\left|k-k_{0}\right| \geq K\left(M-\frac{K-1}{2} M_{1}\right) \geq 2 M_{2},
$$

which is a contradiction. Therefore, for all $k=1, \ldots, K$ and $t \geq T_{\delta}, \xi_{k}(t) \leq M$. One argues similarly to show that $\xi_{k}(t) \geq-M$.

Now, we consider the unperturbed ODE system for the $\left(\xi_{k}\right)_{k=1, \ldots, K}$, that is

$$
\dot{\varpi}=t^{-1} \Phi(\varpi),
$$

where $\varpi=\left(\varpi_{k}\right)_{k=1, \ldots, K}$ and $\Phi: \mathbb{R}^{K} \rightarrow \mathbb{R}^{K}$ is defined by

$$
\left\{\begin{array}{l}
\Phi_{1}(\varpi)=-\gamma_{1}\left(e^{-\left(\varpi_{2}-\varpi_{1}\right)}-1\right), \\
\Phi_{k}(\varpi)=\gamma_{k-1}\left(e^{-\left(\varpi_{k}-\varpi_{k-1}\right)}-1\right)-\gamma_{k}\left(e^{-\left(\varpi_{k+1}-\varpi_{k}\right)}-1\right), \text { for } 2 \leq k \leq K-1, \\
\Phi_{K}(\varpi)=\gamma_{K-1}\left(e^{-\left(\varpi_{K}-\varpi_{K-1}\right)}-1\right) .
\end{array}\right.
$$

This system is studied in $[8]$. Observe that setting $\boldsymbol{e}_{1}=\frac{1}{\sqrt{K}}(1, \ldots, 1)^{T}$, it holds

$$
\text { for all } t, t_{0} \geq T_{\delta}, \quad \frac{1}{K} \sum_{j=1}^{K} \varpi_{j}(t)=\frac{1}{\sqrt{K}}\left(\varpi, e_{1}\right)=\frac{1}{K} \sum_{j=1}^{K} \varpi_{j}\left(t_{0}\right) .
$$

Moreover, $D \Phi(0)$ is the $K \times K$ matrix with entries

$$
\begin{aligned}
& m_{1,1}=-\gamma_{1}, \quad m_{K, K}=-\gamma_{K-1}, \quad m_{k, k}=-\left(\gamma_{k-1}+\gamma_{k}\right), \quad \text { for } k=2, \cdots, K-1, \\
& m_{k, k-1}=\gamma_{k-1}, \quad m_{k, k+1}=\gamma_{k}, \quad m_{k, k^{\prime}}=0, \quad \text { if }\left|k-k^{\prime}\right| \geq 2 .
\end{aligned}
$$

We recall the following properties.

Proposition $5.4([8])$. It holds $D \Phi(0) \boldsymbol{e}_{1}=0$ and

$$
\text { for all } \boldsymbol{x} \in \boldsymbol{e}_{1}^{\perp}, \quad(D \Phi(0) \boldsymbol{x}, \boldsymbol{x}) \leq-\|\boldsymbol{x}\|^{2} \text {. }
$$

Furthermore, for any $M>0$, there exists $C(M)>0$ such that for any $t_{0}>0$, if $\left|\varpi\left(t_{0}\right)\right| \leq M$, then

$$
\text { for all } t \geq t_{0}, \quad\left\|\varpi(t)-\left(\varpi\left(t_{0}\right), e_{1}\right) e_{1}\right\| \leq C(M) t_{0} t^{-1} .
$$

Proof. See [8, Lemma 2.8] for the coercivity (5.19) of $D \Phi(0)$ and [8, Proposition 2.5] for the convergence (5.20) (written there in the variable $\tau=\log t$ ).

Now, we complete the proof of Theorem 1.1 by showing the estimate (5.14).

Proof of (5.14). We summarise what was obtained so far. Let $\boldsymbol{\xi}:=\left(\xi_{k}\right)_{k=1, \ldots, K}$. There exists $C_{\theta}$ such that for $t \geq T_{\delta}$,

(i) $\dot{\boldsymbol{\xi}}=t^{-1} \Phi(\boldsymbol{\xi})+\boldsymbol{r}(t)$ where $\|\boldsymbol{r}(t)\| \leq C_{\theta} t^{-\theta}$,

(ii) $\left|\left(\boldsymbol{\xi}, \boldsymbol{e}_{1}\right)\right| \leq C_{\theta} t^{-\theta+1}$,

(iii) $\|\boldsymbol{\xi}(t)\| \leq \sqrt{K} M$, for $t \geq T_{\delta}$.

Moreover, by (5.19), there exist $\epsilon, C>0$ such that if $\|\boldsymbol{x}\| \leq \epsilon$, then

$$
(\Phi(\boldsymbol{x}), \boldsymbol{x}) \leq-\frac{1}{2}\|\boldsymbol{x}\|^{2}+C\left(\boldsymbol{x}, \boldsymbol{e}_{1}\right)^{2} .
$$

Step 1. We claim that there exists $T_{\epsilon} \geq T_{\delta}$ such that

$$
\text { for all } t \geq T_{\epsilon}, \quad\|\boldsymbol{\xi}(t)\| \leq \epsilon .
$$

Let $L=4 \epsilon^{-1} C(M)+1$, where $C(M)$ given by Proposition 5.4. Set

$$
A=3+\sup \{\|D \Phi(\boldsymbol{x})\|:\|\boldsymbol{x}\| \leq \max (C(M), \sqrt{K} M)\} .
$$


By (i) and (ii), we fix $t_{0}$ large enough such that

$$
\sup _{t \geq t_{0}}\left(t \sup _{s \geq t}\|\boldsymbol{r}(s)\|\right) \leq \frac{\epsilon}{4} L^{-A} \quad \text { and } \quad t_{0}^{-\theta+1} \leq \frac{\epsilon}{2} C_{\theta}^{-1} .
$$

For any $t_{1} \geq t_{0}$, we denote $\varpi_{t_{1}}$ the solution of (5.18) with data $\varpi_{t_{1}}\left(t_{1}\right)=\boldsymbol{\xi}\left(t_{1}\right)$ at time $t_{1}$. On the one hand, by standard Gronwall estimates, it holds for any $t \geq t_{1}$,

$$
\left\|\boldsymbol{\xi}(t)-\varpi_{t_{1}}(t)\right\| \leq t^{A} \int_{t_{1}}^{t} s^{-A}\|\boldsymbol{r}(s)\| \mathrm{d} s \leq t^{A} t_{1}^{-A+1} \sup _{s \geq t_{1}}\|\boldsymbol{r}(s)\| .
$$

Let $t=L t_{1}$, using the definition of $t_{0}$, we obtain

$$
\left\|\boldsymbol{\xi}\left(L t_{1}\right)-\varpi_{t_{1}}\left(L t_{1}\right)\right\| \leq L^{A}\left(t_{1} \sup _{s \geq t_{1}}\|\boldsymbol{r}(s)\|\right) \leq \frac{\epsilon}{4} .
$$

On the other hand, using (5.20), (ii) and the definition of $t_{0}$,

$$
\left\|\varpi_{t_{1}}\left(L t_{1}\right)\right\| \leq\left\|\left(\boldsymbol{\xi}\left(t_{1}\right), \boldsymbol{e}_{1}\right) \boldsymbol{e}_{1}\right\|+C(M) t_{1}\left(L t_{1}\right)^{-1} \leq \frac{3}{4} \epsilon .
$$

Let $T_{\epsilon}=L t_{0}$, from (5.23) and (5.24), we obtain (5.22).

Step 2. Using (i), (ii), (5.21) and (5.22), we infer that for $t \geq T_{\epsilon}$,

$$
\begin{aligned}
\frac{d}{d t}\|\boldsymbol{\xi}\|^{2} & =2 t^{-1}(\Phi(\boldsymbol{\xi}), \boldsymbol{\xi})+2(r, \boldsymbol{\xi}) \\
& \leq-t^{-1}\|\boldsymbol{\xi}\|^{2}+O\left(t^{1-2 \theta}\right)+O\left(t^{-\theta}\|\boldsymbol{\xi}\|\right) \\
& \leq-\left(\theta-\frac{1}{2}\right) t^{-1}\|\boldsymbol{\xi}\|^{2}+O\left(t^{1-2 \theta}\right) .
\end{aligned}
$$

A direct integration and $1<\theta<3 / 2$ yield, for some $C>0$,

$$
\|\boldsymbol{\xi}(t)\|^{2} \leq\left(\frac{T_{\epsilon}}{t}\right)^{\theta-\frac{1}{2}}\left\|\boldsymbol{\xi}\left(T_{\epsilon}\right)\right\|^{2}+C t^{-2 \theta+2} \lesssim t^{-2 \theta+2},
$$

which is (5.14).

\section{Construction of multi-Solitary Waves}

In this Section, we prove Theorem [1.3, adapting arguments from 6, Section 4]. Here, $\mathcal{B}_{H^{1} \times L^{2}}(\delta)$ denotes the open ball of $H^{1} \times L^{2}$ of center 0 and radius $\delta$ and $\mathcal{B}_{\mathbb{R}^{K}}(\delta)$ (respectively, $\overline{\mathcal{B}}_{\mathbb{R}^{K}}(\delta)$ ) denotes the open ball (respectively, closed ball) of $\mathbb{R}^{K}$ of center 0 and radius $\delta$. Last, $\mathcal{S}_{\mathbb{R}^{K}}(\delta)$ denotes the sphere of $H^{1} \times L^{2}$ of center 0 and radius $\delta$. We also use the notation from $\$ 3$ and set

$$
\beta:=\frac{1}{2 \sqrt{\alpha^{2}+\nu_{0}^{2}}}=\left\langle\vec{Y}^{+}, \vec{Z}^{+}\right\rangle^{-1}>0 .
$$

We recall the following preliminary result (for the proof, see [6, Lemma 4.1]).

Lemma 6.1. Let $\left(z_{k}, \ell_{k}\right)_{k=1, \ldots, K} \in \mathbb{R}^{2 K}$ be such that

$$
r=\min \left(z_{k+1}-z_{k}, k=1, \ldots, K-1\right)
$$

is large enough. There exist linear maps

$$
B: \mathbb{R}^{K} \rightarrow \mathbb{R}^{K}, \quad V: \mathbb{R}^{K} \rightarrow \mathbb{R}^{K}
$$

smooth in $\left(z_{k}, \ell_{k}\right)_{k=1, \ldots, K}$, satisfying

$$
\|B-\beta \operatorname{Id}\| \lesssim e^{-\frac{1}{2} r}, \quad\left\|V_{j}\right\| \lesssim e^{-\frac{1}{2} r},
$$

and such that the function $W(\boldsymbol{a}): \mathbb{R} \rightarrow \mathbb{R}$ defined by

$$
W(\boldsymbol{a})(x):=\sum_{k=1}^{K}\left\{B_{k}(\boldsymbol{a}) Y_{k}(x)+V_{k}(\boldsymbol{a}) \partial_{x} Q_{k}(x)\right\},
$$


for any $\boldsymbol{a}=\left(a_{1}, \ldots, a_{k}\right)$, satisfies, for all $k=1, \ldots, K$,

$$
\left\langle W(\boldsymbol{a}), \partial_{x} Q_{k}\right\rangle=0, \quad\left\langle W(\boldsymbol{a}), Y_{k}\right\rangle=\beta a_{k} .
$$

In particular, setting

$$
\vec{W}(\boldsymbol{a})=\left(\begin{array}{c}
W(\boldsymbol{a}) \\
\nu^{+} W(\boldsymbol{a})
\end{array}\right) \quad \text { it holds } \quad\left\langle\vec{W}(\boldsymbol{a}), \vec{Z}_{k}^{+}\right\rangle=a_{k} .
$$

The next proposition and the invariance by translation of (1.1) imply Theorem 1.3.

Proposition 6.2. Let $K \geq 2$ and $\sigma= \pm 1$. For $\delta>0$ small enough, let any

$$
\left\{\begin{array}{l}
\left(\ell_{k}(0)\right)_{k=1, \ldots, K} \in \mathcal{B}_{\mathbb{R}^{K}}(\delta), \\
\left(z_{k}(0)\right)_{k=1, \ldots, K} \in \mathbb{R}^{K} \\
\quad \text { satisfying } \min \left\{z_{k+1}(0)-z_{k}(0), k=1, \ldots, K-1\right\}>5|\log \delta|, \\
\vec{\varepsilon}_{\perp}(0) \in \mathcal{B}_{H^{1} \times L^{2}}(\delta) \\
\quad \text { satisfying (3.16) and }\left\langle\vec{\varepsilon}_{\perp}(0), \vec{Z}_{k}^{+}(0)\right\rangle=0 \text { for } k=1, \ldots, K .
\end{array}\right.
$$

There exists $\boldsymbol{a}_{\sharp}^{+}(0)=\left(a_{\sharp, k}^{+}(0)\right)_{k=1, \ldots, K} \in \overline{\mathcal{B}}_{\mathbb{R}^{K}}\left(\delta^{\frac{5}{4}}\right)$ such that the solution $\vec{u}_{\sharp}$ of (1.1) with the initial data

$$
\vec{u}_{\sharp}(0)=\sigma \sum_{k=1}^{K}(-1)^{k}\left(Q\left(\cdot-z_{k}(0)\right), 0\right)+\vec{W}\left(\boldsymbol{a}_{\sharp}^{+}(0)\right)+\vec{\varepsilon}_{\perp}(0)
$$

is global and satisfies (1.6) where, for $k=1, \ldots, K, z_{k}=\bar{y}_{k}+y_{\sharp}+O\left(t^{-\theta+1}\right)$, for some $y_{\sharp} \in \mathbb{R}$ and $\bar{y}_{k}$ being defined in (5.11).

Remark 6.3. From the proof of Proposition 6.2 there exist even solutions of (1.1) with any odd number $K \geq 3$ of solitary waves.

Proof. Given $\boldsymbol{a}^{+}(0)=\left(a_{k}^{+}(0)\right)_{k=1, \ldots, K} \in \overline{\mathcal{B}}_{\mathbb{R}^{K}}\left(\delta^{\frac{5}{4}}\right)$, we consider the solution $\vec{u}(t)$ of (1.1) with initial data

$$
\vec{u}(0)=\sigma \sum_{k=1}^{K}(-1)^{k}\left(Q\left(\cdot-z_{k}(0)\right), 0\right)+\vec{W}\left(\boldsymbol{a}^{+}(0)\right)+\vec{\varepsilon}_{\perp}(0) .
$$

Decomposition. For any $t \geq 0$ such that $\vec{u}(t)$ is defined and satisfies (3.14), we consider its decomposition according to Lemma 3.2. Following $\$ 3.4$, we introduce the notation $y_{k}(k=1, \ldots, K), \mathcal{N}, \mathcal{M}$ and

$$
F=\sum_{k=1}^{K-1} e^{-\left(y_{k+1}-y_{k}\right)}, \quad R=\frac{1}{F} \exp \left(-3 \lambda^{-1} \mathcal{M}\right)
$$

(due to the choice of signs in the decomposition of $\vec{u}(0), F=F_{-}$and $F_{+}=0$ ). Note that by the properties of the function $W$ in Lemma 6.1 and the orthogonality properties (3.16) of $\vec{\varepsilon}_{\perp}(0)$ assumed in (6.1), the initial data $\vec{u}(0)$ is modulated. Indeed, $\left(z_{k}(0), \ell_{k}(0)\right)_{k=1, \ldots, K}$ and

$$
\vec{\varepsilon}(0)=\vec{W}\left(\boldsymbol{a}^{+}(0)\right)+\vec{\varepsilon}_{\perp}(0),
$$

are the parameters of the decomposition of $\vec{u}(0)$. In particular, it holds from (6.1)

$$
\mathcal{N}(0) \lesssim \delta, \quad F(0) \lesssim \delta^{2} .
$$

Moreover, by Lemma 6.1 for $k=1, \cdots, K$, it holds

$$
\left\langle\vec{\varepsilon}(0), \vec{Z}_{k}^{+}(0)\right\rangle=\left\langle\vec{W}\left(\boldsymbol{a}^{+}(0)\right), Z_{k}^{+}\right\rangle=a_{k}^{+}(0),
$$

which is consistent with the definition of $a_{k}^{+}$in (v) of Lemma 3.2.

Bootstrap estimates. We introduce the following bootstrap estimates

$$
\mathcal{N} \leq \delta^{\frac{3}{4}}, \quad F \leq \delta^{\frac{3}{2}}, \quad b \leq \delta^{\frac{5}{2}}
$$


and we set $T_{*}=\sup \{t \in[0, \infty)$ such that $(6.2)$ holds on $[0, t]\} \geq 0$.

Estimates on the damped components. The estimate on $\mathcal{N}$ is strictly improved on $\left[0, T_{*}\right]$ as in the proof of Proposition 3.12 In particular, $\mathcal{N} \lesssim \delta$ on $\left[0, T_{*}\right]$.

Estimate on the distance. Inequality (3.37) rewrites

$$
\frac{\mathrm{d} R}{\mathrm{~d} t} \geq\left(\lambda+\frac{2}{\lambda F}\left(\mathcal{N}^{2}-F^{\theta}\right)\right) \exp \left(-3 \lambda^{-1} \mathcal{M}\right) .
$$

Now for $t \in\left[0, T_{*}\right]$, in view of (6.2), $\exp \left(-3 \lambda^{-1} \mathcal{M}\right)=1+O\left(\mathcal{N}^{2}\right)=1+O\left(\delta^{3 / 2}\right)$ and $F^{\theta-1}=O\left(\delta^{3(\theta-1) / 2}\right) \leq \frac{1}{6 \lambda^{2}}$ so that

$$
\frac{\mathrm{d} R}{\mathrm{~d} t} \geq \frac{2}{3} \lambda\left(1-C \delta^{3 / 2}\right) \geq \frac{\lambda}{2} .
$$

Integrating on $[0, t] \subset\left[0, T_{*}\right]$, it holds $R(t) \geq R(0)+\frac{\lambda}{2} t$. Hence, as we also have $\exp \left(3 \lambda^{-1} \mathcal{M}\right)=1+O\left(\delta^{3 / 2}\right)$ and $R(0)^{-1} \leq 2 F(0) \lesssim \delta^{2}$,

$$
F=\frac{1}{R} \exp \left(3 \lambda^{-1} \mathcal{M}\right) \leq \frac{1}{R(0)+\frac{\lambda}{2} t}\left(1+O\left(\delta^{3 / 2}\right)\right) \lesssim \delta^{2} .
$$

This strictly improves the estimate of $F$ in (6.2), and as in the proof of Proposition 5.1, we also obtain the decay $F \lesssim t^{-1}$.

Transversality condition. From (3.33) and $\mathcal{N} \lesssim \delta$, we observe that for any time $t \in\left[0, T_{*}\right]$ where it holds $b(t)=\delta^{\frac{5}{2}}$, we have

$$
\frac{\mathrm{d}}{\mathrm{d} t} b(t) \geq 2 \nu^{+} b(t)-C \delta^{3} \geq 2 \nu^{+} \delta^{\frac{5}{2}}-C \delta^{3} \geq \nu^{+} \delta^{\frac{5}{2}}>0,
$$

for $\delta>0$ small enough. This transversality condition is enough to justify the existence of at least a point $\boldsymbol{a}_{\sharp}^{+}(0) \in \overline{\mathcal{B}}_{\mathbb{R}^{K}}\left(\delta^{\frac{5}{4}}\right)$ such that $T_{*}=\infty$.

Indeed, for the sake of contradiction assume that for all $\boldsymbol{a}^{+}(0) \in \overline{\mathcal{B}}_{\mathbb{R}^{K}}\left(\delta^{\frac{5}{4}}\right)$, it holds $T_{*}<\infty$. Then, a contradiction follows from the following observations (see for instance more details in [5] or in [7, Section 3.1]).

Continuity of $T_{*}$. The above transversality condition implies that the map

$$
\boldsymbol{a}^{+}(0) \in \overline{\mathcal{B}}_{\mathbb{R}^{K}}\left(\delta^{\frac{5}{4}}\right) \mapsto T_{*} \in[0, \infty)
$$

is continuous and that $T_{*}=0$ for $\boldsymbol{a}^{+}(0) \in \mathcal{S}_{\mathbb{R}^{K}}\left(\delta^{\frac{5}{4}}\right)$.

Construction of a retraction. As a consequence, the map

$$
\boldsymbol{a}^{+}(0) \in \overline{\mathcal{B}}_{\mathbb{R}^{K}}\left(\delta^{\frac{5}{4}}\right) \mapsto \boldsymbol{a}^{+}\left(T_{*}\right) \in \mathcal{S}_{\mathbb{R}^{K}}\left(\delta^{\frac{5}{4}}\right)
$$

is continuous and its restriction to the sphere $\mathcal{S}_{\mathbb{R}^{K}}\left(\delta^{\frac{5}{4}}\right)$ is the identity.

This is a contradiction with the no retraction theorem for continuous maps from the ball to the sphere.

At this point, we have proved the existence of $\boldsymbol{a}_{\sharp}^{+}(0) \in \overline{\mathcal{B}}_{\mathbb{R}^{K}}\left(\delta^{\frac{5}{4}}\right)$, associated with a global solution $\vec{u}_{\sharp} \in \mathcal{C}\left([0, \infty), H^{1}\right)$ of (1.1), which also satisfies (6.2) for all $t \geq 0$ (and $F=F_{-}, F_{+}=0$ ). Applying the results of Section 5 to $\vec{u}_{\sharp}$, we infer that $\mathcal{N} \lesssim t^{-1}$ (so that (1.6) holds) and that there exists $y_{\sharp} \in \mathbb{R}$ with, for $k=1, \ldots, K$,

$$
y_{k}=\bar{y}_{k}+y_{\sharp}+O\left(t^{-\theta+1}\right)
$$

As $z_{k}=y_{k}-\frac{\ell_{k}}{2 \alpha}$ and $\left|\ell_{k}\right| \leq \mathcal{N} \lesssim t^{-1}, u_{\sharp}$ has the requested properties and the proof is complete.

\section{REFERENCES}

[1] N. Burq, G. Raugel and W. Schlag, Long time dynamics for damped Klein-Gordon equations, Ann. Sci. Éc. Norm. Supér., 50 (2017), 1447-1498.

[2] T. Cazenave, Uniform estimates for solutions of nonlinear Klein-Gordon equations, Journal of Functional Analysis, 60 (1985), 36-55. 
[3] T. Cazenave and A. Haraux, An introduction to semilinear evolution equations. Oxford Lecture Series in Mathematics and its Applications, 13. The Clarendon Press, Oxford University Press, New York, 1998.

[4] R. Côte, On the soliton resolution for equivariant wave maps to the sphere, Communications on Pure and Applied Mathematics, 68 (2015), 1946-2004.

[5] R. Côte, Y. Martel and F. Merle, Construction of multi-soliton solutions for the $L^{2}$ supercritical gKdV and NLS equations, Rev. Mat. Iberoamericana 27 (2011), 273-302.

[6] R. Côte, Y. Martel, X. Yuan and L. Zhao. Description and classification of 2-solitary waves for nonlinear damped Klein-Gordon equations. Preprint arXiv:1908.09527

[7] R. Côte and C. Muñoz, Multi-solitons for nonlinear Klein-Gordon equations, Forum of Mathematics, Sigma. 2 (2014), 38 pp.

[8] R. Côte and H. Zaag, Construction of a multisoliton blowup solution to the semilinear wave equation in one space dimension, Commun. Pure Appl. Math., 66 (2013), 1541-1581.

[9] T. Duyckaerts, C. E. Kenig and F. Merle, Classification of radial solutions of the focusing, energy-critical wave equation, Cambridge J. Math., 1 (2013), 75-144.

[10] T. Duyckaerts, H. Jia, C. E. Kenig and F. Merle, Soliton resolution along a sequence of times for the focusing energy critical wave equation, Geom. Funct. Anal., 27 (2017), 798-862.

[11] E. Feireisl, Finite energy travelling waves for nonlinear damped wave equations, Quart. Appl. Math., 56 (1998), 5570 .

[12] J. Jendrej, Dynamics of strongly interacting unstable two-solitons for generalized Korteweg-de Vries equations. Preprint arXiv:1802.06294

[13] P. L. Lions, Solutions of Hartree-Fock equations for Coulomb systems, Comm. Math. Phys., 109 (1987), 33-97.

[14] P. L. Lions, On positive solutions of semilinear elliptic equations in unbounded domains, Nonlinear diffusion equations and their equilibrium states, II (Berkeley, CA, 1986), 85-122, Math. Sci. Res. Inst. Publ., 13, Springer, New York, 1988.

[15] Z. Li and L. Zhao, Asymptotic decomposition for nonlinear damped Klein-Gordon equations, Preprint arXiv:1511.00437.

[16] F. Merle and H. Zaag, Existence and classification of characteristic points at blow-up for a semilinear wave equation in one space dimension, Amer. J. Math., 134 (2012) n. 3, 581-648.

[17] T. V. Nguyen, Strongly interacting multi-solitons with logarithmic relative distance for the gKdV equation, Nonlinearity, 30(12):4614, 2017.

[18] T. V. Nguyen, Existence of multi-solitary waves with logarithmic relative distances for the NLS equation, C. R. Math. Acad. Sci. Paris, 357 (2019), 13-58.

[19] A. Pazy, Semigroups of linear operators and applications to partial differential equations, Appl. Math. Sci., vol. 44, Springer-Verlag, New York, Berlin, Heidelberg, Tokyo, 1983.

IRMA UMR 7501, Université de Strasbourg, CNRS, F-67000 Strasbourg, France

E-mail address: cote@math.unistra.fr

CMls, École polytechnique, CNRS, Institut Polytechnique de Paris, 91128 Palaiseau Cedex, France

E-mail address: yvan.martel@polytechnique.edu

CMls, École polytechnique, CNRS, Institut Polytechnique de Paris, 91128 Palaiseau Cedex, France

E-mail address: xu.yuan@polytechnique.edu 